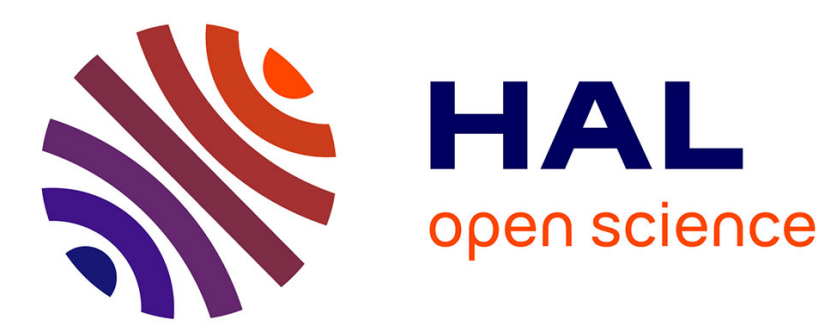

\title{
Calibration of anisotropic plasticity models using a biaxial test and the virtual fields method
}

\author{
J.M.P. Martins, A. Andrade-Campos, S. Thuillier
}

\section{To cite this version:}

J.M.P. Martins, A. Andrade-Campos, S. Thuillier. Calibration of anisotropic plasticity models using a biaxial test and the virtual fields method. International Journal of Solids and Structures, 2019, 172, pp.21 - 37. 10.1016/j.ijsolstr.2019.05.019 . hal-03485827

\section{HAL Id: hal-03485827 \\ https://hal.science/hal-03485827}

Submitted on 20 Dec 2021

HAL is a multi-disciplinary open access archive for the deposit and dissemination of scientific research documents, whether they are published or not. The documents may come from teaching and research institutions in France or abroad, or from public or private research centers.
L'archive ouverte pluridisciplinaire HAL, est destinée au dépôt et à la diffusion de documents scientifiques de niveau recherche, publiés ou non, émanant des établissements d'enseignement et de recherche français ou étrangers, des laboratoires publics ou privés.

\section{다)(1) $(5$}

Distributed under a Creative Commons Attribution - NonCommerciall 4.0 International 


\title{
Calibration of anisotropic plasticity models using a biaxial test and the virtual fields method
}

\author{
J.M.P. Martins ${ }^{\mathrm{a}, \mathrm{b}, *}$, A. Andrade-Campos ${ }^{\mathrm{a}}$, S. Thuillier ${ }^{\mathrm{b}}$ \\ ${ }^{a}$ Centre for Mechanical Technology and Automation (TEMA), GRIDS Research Unit, Mechanical Engineering Department, \\ University of Aveiro \\ ${ }^{b}$ Univ. Bretagne Sud, UMR CNRS 6027, IRDL, F-56100 Lorient, France
}

\begin{abstract}
The aim of the present study is to explore the combination of a biaxial test with a cruciform specimen and the virtual fields method to develop an efficient strategy for simultaneous identification of material parameters related with hardening and anisotropy in plasticity. In a first step, three cruciform geometries are evaluated as potential candidates to generate an experimental database for the calibration of the classical Hill'48 yield criterion and Swift's hardening law. In a second step, the geometry with the best results is used to calibrate YLD2000-2d yield criterion and Swift's hardening law. Numerical results are used as virtual experimental full-field measurements, which allows the comparison of the identified solution with the input material parameters. The accuracy of the identified material parameters is thoroughly assessed through the analysis of flow stress curve evolution, normalised yield stresses and plastic anisotropy coefficients, and for the last step, yield locus prediction. The results show the potential of this combination to identify simultaneously the material parameters related to hardening and anisotropy with a single test.
\end{abstract}

Keywords: Anisotropic plasticity, Cruciform test, Full-field measurements, Parameter identification, Virtual fields method

\section{Introduction}

The development of accurate and efficient models for the plastic behaviour of sheet metals has been crucial to improve components performance and to validate manufacturing processes through numerical simulation. In the last years, efforts have been oriented to provide models with more realistic descriptions 5 of phenomena such as hardening and anisotropy [1]. In the case of phenomenological models, which are usually preferred for Finite Element (FE) simulations, the improvement in accuracy has been associated to increased flexibility in the mathematical formulation, which has consequently led to an increase in the number of material parameters of the most recent models [2]. The increase in the number of parameters brought additional challenges for the identification of these parameters (or calibration process). The main challenge has been to gather required data to properly identify the material parameters, which usually implies a time-consuming experimental campaign [3]. Furthermore, the required data should be representative of the stress and strain states of the application that is intended to be simulated $[4,5]$. These challenges have hampered the widespread use of more recent and accurate models [1].

The calibration process is the first step before any practical application of a constitutive model and it is directly related with its accuracy [6]. Generally, a constitutive model to predict the plastic flow of sheet metals is defined by a hardening law and a yield criterion, within the framework of the associated flow rule. The calibration of both components is usually performed separately. For example, in the case of isotropic

\footnotetext{
* Corresponding author

Email addresses: joao.martins52@ua.pt (J.M.P. Martins), gilac@ua.pt (A. Andrade-Campos), sandrine.thuillier@univ-ubs.fr (S. Thuillier)
} 
hardening, Swift's law [7] is traditionally calibrated by adjusting its prediction to stress-strain curves from uniaxial tensile tests or, when desired to extend the strain range, from hydraulic bulge tests [8]. Regarding

20 the yield criterion, modelling the anisotropic plastic behaviour of sheet metals has been a very active field and consequently, a large spectrum of yield criteria with different levels of complexity is available now. However, the criterion proposed by Hill [9] (Hill'48 yield criterion) is still referred to as the most used yield criterion, a fact directly connected to the simplicity of both the formulation and the calibration process. In plane stress condition, this criterion depends only on 4 parameters which can be analytically calculated 25 using the plastic anisotropy coefficient (or Lankford's parameter) in three different orientations i.e. rolling, diagonal and transverse ones, and the initial yield stress in the rolling direction. To collect this data, three uniaxial tensile tests at the three orientations are required. Nevertheless, its formulation lacks flexibility which restricts the representation of the mechanical behaviour of certain materials [10], and also cannot predict both the initial yield stresses and the plastic anisotropy coefficients with the same accuracy [11]. Therefore, more flexible formulations have been proposed. Among others, the criterion proposed by Barlat et al. [12] (YLD2000-2d) can be given as example. The calibration of this yield criterion requires more information than Hill'48 yield criterion, namely, the plastic anisotropy coefficients and the yield stresses in different orientations of the sheet and also, the plastic anisotropic coefficient and yield stress from an equi-biaxial test. Moreover, the material parameters are identified using an optimisation procedure.

The examples presented above illustrate classical calibration procedures for the different components of a constitutive model. Nevertheless, the combination of full-field measurement techniques, such as digital image correlation (DIC), and the inverse methods developed to take full advantage of such data can simplify the calibration of classical plasticity models $[13,14]$. In this combined strategy, complex specimen geometries can be used in order to produce heterogeneous displacements/strain fields. An ideal geometry should produce 40 heterogeneous displacement/strain fields that contain the required experimental data to calibrate a model and thereby, reduce the number of tests of the experimental campaign. Accordingly, the cruciform geometry has been investigated over the years [15]. This type of geometry in biaxial tension is known for producing strain paths ranging from biaxial tension, at the centre of the specimen, to uniaxial tension, in the arms of the specimen [16, 17]. Moreover, Zhang et al. [16] through numerical analysis, showed that the prediction of the strain distribution in the central area of the specimen is dependent of the yield criterion selected.

The studies conducted on the biaxial test with the cruciform geometry are often based on inverse methods of a FEMU-based type. This type of inverse methods determines the best set of material parameters through the minimisation of a function representing the gap between numerical and experimental results. This function can be built based on strain or displacement fields or force values, or it can be a combination of these quantities. In the framework of plasticity, a thorough review on the combination of the biaxial test with the cruciform geometry and FEMU-based inverse methods can be found in [15]. As an example of such studies, it can be mentioned the work of Zhang et al. [16]. These authors proposed a geometry for a cruciform specimen and succeeded to identify the material parameters of Bron and Besson yield criterion [18] (criterion with a total of 13 parameters). The function to be minimised was built using the major and minor strain

55 distributions along a diagonal path in the central area of the specimen captured for single time instant of the test. More recently, Coopieters et al. [19] used a perforated cruciform specimen [20] to study the influence of boundary conditions, number of time instants and settings of DIC system on the identification of Hill'48 criterion's parameters using a FEMU inverse method. This study also reveals that such an inverse approach can give better results than a conventional identification procedure for Hill' 48 yield criterion.

Although FEMU-based inverse methods can be considered a feasible tool for parameter identification, these are excessively time-consuming, a drawback attributed to the FE simulations required within the iterative process of identification. The virtual fields method (VFM) is an alternative to overcome this drawback [14]. This inverse method relies on the principle of virtual work and on the choice of a set of virtual fields [21]. In the framework of plasticity, the most recent applications of the VFM have been the 65 simultaneous calibration of isotropic hardening and anisotropic yield criteria [22, 23], the calibration of kinematic hardening law [24] and a distortional hardening model [25]. Rossi et al. [22] used a notched specimen to perform the simultaneous calibration of Swift's law and two criteria, namely i.e. Hill'48 yield criterion and YLD2000-2d yield criterion [12]. The obtained results were reasonable, although the authors suggested that a different specimen could improve the results. Marek et al. [23] focused on the validation of 
a new strategy to automatically determine an optimal set of virtual fields for the calibration process. The results have been improved, but the same notched specimen was used.

The purpose of the present study is to explore the combination of the VFM and a cruciform specimen tested in biaxial tension for the simultaneous calibration of an isotropic hardening law and an anisotropic yield criterion. This combination has already been tested, but for the framework of hyperelasticity [26]. In that case, the number of material parameters was just two, corresponding to the Mooney's hyperelastic model in plane stress conditions, and a linear version of the VFM was used [21]. The good results obtained proved the feasibility of this combination. Nevertheless, any study on this combination has been performed in the framework of anisotropic plasticity. The present study is performed with virtual experimental data and the reference parameters are known a priori, which allows to compare directly with the identification results and evaluate the quality of the information obtained from the test. In this first analysis, the evaluation of DIC settings and noise effect are out of the scope, though it will be required in a further analysis. The outline of the article is as follows. Section 2 presents the VFM and a set of virtual fields that can be used with a cruciform specimen submitted to biaxial tension. Section 3 introduces the constitutive models addressed in this study, namely Swift's hardening law and the yield criteria Hill'48 and YLD2000-2d. In Section 4, three cruciform geometries are evaluated as potential candidates to combine with the VFM. A thorough evaluation of the generated strain and stress fields in these geometries is performed. Section 5 provides a detailed discussion regarding the identification results obtained, namely a comparison of the three cruciform geometry and the performance of the best geometry in the calibration of YLD2000-2d and Swift's law.

\section{The virtual fields method}

\subsection{Theoretical background}

The VFM is an inverse method for parameter identification based on full-field measurements which relies on the principle of virtual work, written in the framework of large strains in this study. This large strains formulation has been firstly applied to the calibration of hyperelastic models by Promma et al. [26] and subsequently, adopted in several studies for the calibration of plasticity models [22, 23, 27]. Nevertheless, other formulations can be found in [21, 28, 29].

First, reference and current configurations of a continuum solid body $\Omega$ must be distinguished. Consider the reference or undeformed configuration $\Omega_{0}$ occupied by the solid body at the beginning of the deformation process (time instant $t=0$ ) and delimited by $\Gamma_{0}$. Also, consider that the boundary $\Gamma_{0}$ is composed of two sub-boundaries $\Gamma_{0}^{u}$ and $\Gamma_{0}^{f}$, such that $\Gamma_{0}=\Gamma_{0}^{u} \cup \Gamma_{0}^{f}$ and $\Gamma_{0}^{u} \cap \Gamma_{0}^{f}=\emptyset . \Gamma_{0}^{u}$ corresponds to the boundary with eribed displacements and $\Gamma_{0}^{f}$ the boundary with prescribed force. Moreover, the current or deformed configuration $\Omega_{t}$ is assumed as the region occupied by $\Omega$ after the deformation process at the time instant $t$. The position of a particle in the reference configuration $\Omega_{0}$ is described by $\mathbf{X}$ relative to a reference frame and $\mathbf{x}(\mathbf{X}, t)$ gives the position of this same particle in the current configuration $\Omega_{t}$. The displacement field which relates the position of this particle in the reference configuration and its position in the current configuration is given by $\mathbf{u}(\mathbf{X}, t)=\mathbf{x}(\mathbf{X}, t)-\mathbf{X}$.

Assuming quasi-static conditions and neglecting body forces, the principle of virtual work can be expressed in the reference configuration as:

$$
-\underbrace{\int_{\Omega_{0}} \mathbf{P}: \operatorname{grad} \mathbf{U}^{*} \mathrm{~d} V}_{W_{\text {int }}}+\underbrace{\int_{\Gamma_{0}^{f}} \overline{\mathbf{T}} \cdot \mathbf{U}^{*} \mathrm{~d} S}_{W_{\text {ext }}}=0 .
$$

Here, $\mathbf{P}$ is the first Piola-Kirchhoff stress tensor, $\mathbf{U}^{*}$ is the virtual displacement vector defined in the reference configuration $\mathbf{X}$, as well as grad $\mathbf{U}^{*}[30] . \overline{\mathbf{T}}$ is the first Piola-Kirchhoff stress vector prescribed on the boundary $\Gamma_{0}^{f}$ with reference surface area $S$. Similarly, the first integral of Eq. 1 has to be computed over the reference volume $V$. The virtual displacement field $\left(\mathbf{U}^{*}\right)$ must be a continuous and differentiable function and it is assumed, for a matter of convenience [21,30], that it vanishes on the boundary of applied displacements $\Gamma_{0}^{u}$ (condition 1). 
It is also useful to establish the link between reference and current configurations. Thus, the first PiolaKirchhoff stress tensor can be defined in terms of the Cauchy stress tensor $\boldsymbol{\sigma}$ as

$$
\mathbf{P}=\operatorname{det}(\mathbf{F}) \boldsymbol{\sigma} \mathbf{F}^{-\mathrm{T}},
$$

where $\operatorname{det}(\mathbf{F})$ is the determinant of the deformation gradient $\mathbf{F}$ and $\mathbf{F}^{-\mathrm{T}}$ is the transpose of its inverse. The deformation gradient can be defined as

$$
\mathbf{F}=\frac{\partial \mathbf{x}}{\partial \mathbf{X}}=\mathbf{I}+\frac{\partial \mathbf{u}}{\partial \mathbf{X}}
$$

where $\mathbf{I}$ is the second order unity tensor.

According to Eq. 1, the principle of virtual work states, for a body in equilibrium and for a continuous virtual field, that the internal virtual work $W_{\text {int }}$ equals the external virtual work $W_{\text {ext }}$ and this should be satisfied for every time instant $t$. The computation of the internal virtual work is therefore a key point that requires a constitutive model to establish the link between the stress field and the strain field. In the framework of elasto-plasticity, the relation between the stress field and the strain field is non-linear and history-dependent or path-dependent. Elasto-plastic constitutive models are commonly formulated in terms of the Cauchy stress tensor $\boldsymbol{\sigma}$. Therefore, the stress field corresponding to a defined time instant $t$ is usually a function of the material parameters (consider $\boldsymbol{\xi}$ as the vector that gathers all material parameters) and the history of deformation, here denoted by $\left.\varepsilon\right|_{0 \rightarrow t}$, and can be defined as

$$
\boldsymbol{\sigma}^{t}=\boldsymbol{\sigma}^{t}\left(\boldsymbol{\xi},\left.\boldsymbol{\varepsilon}\right|_{0 \rightarrow t}\right)
$$

The principle of virtual work is the foundation for the VFM. In non-linear cases, to solve the inverse problem and find the best set of material parameters, the main idea behind the VFM is the minimisation of the difference between internal and external virtual work. The objective function is written in a least-square form as

$$
\varphi(\boldsymbol{\xi})=\left(W_{\text {int }}(\boldsymbol{\xi})-W_{\text {ext }}\right)^{2} .
$$

In practice, the internal and external virtual works are computed from measured quantities, namely the displacement fields and the resultant of the applied loads. The internal virtual work results from the reconstruction of the stress field, which in turn is performed using the strain fields and the adopted constitutive model. The strain fields are computed from the measured displacement fields $\mathbf{u}$ by means of the deformation gradient. Using the theorem of polar decomposition, the deformation gradient can be decomposed as

$$
\mathbf{F}=\mathbf{V R}
$$

with $\mathbf{V}$ the left Cauchy stretch tensor and $\mathbf{R}$ is the orthogonal rotation tensor. The strain field can be computed for each time instant as the Hencky's strain tensor

$$
\varepsilon=\ln \mathbf{V} .
$$

Moreover, the computation of the stress field is conventionally expressed in a local material frame free of rigid body rotations, thus the strain tensor in this local co-rotational frame can be computed as

$$
\hat{\varepsilon}=\mathbf{R}^{\mathrm{T}} \boldsymbol{\varepsilon} \mathbf{R} .
$$

Full-field measurements are generally presented as a discrete number of measurements points on the surface of the specimen, and to apply the VFM, these points are usually fitted to a mesh of elements. Strain and stress tensors are then calculated at the centroid of these elements and become representative of the average strain and stress field in a specific element. This calculation can be performed, for example, by means of shape functions. This discrete form of the data allows to approximate the integral of the internal virtual work by a discrete sum as follows

$$
W_{\text {int }}(\boldsymbol{\xi}, t)=\sum_{i=1}^{n_{\mathrm{e}}} \mathbf{P}(\boldsymbol{\xi}, t): \operatorname{grad} \mathbf{U}^{*} A_{i} e_{i} .
$$


where $n_{\mathrm{e}}$ represents the number of elements and $A_{i}$ and $e_{i}$ represent the area and the thickness of each element. The elemental area $A_{i}$ and elemental thickness $e_{i}$ do not depend on time, these quantities refer to the reference configuration $\Omega_{0}$. This is one of the advantages of formulating the principle of virtual work in the reference configuration.

The formulation presented can be applied to three dimensional data. Indeed, there are a few attempts to use it with three dimensional measurements, such as $[27,31,32]$. Nevertheless, as mentioned before, full-field measurements are commonly available just on the surface of the specimen. To overcome the lack of information through-thickness, plane stress conditions are generally assumed which is reasonable when the specimen thickness is much smaller compared to the other dimensions. Nevertheless, the determination of the first Piola-Kirchhoff stress tensor requires additional assumptions [23], namely the determinant of the deformation gradient $\operatorname{det}(\mathbf{F})$, which can be seen as the local ratio of current to reference volume [33]. The determinant of the deformation gradient can be determined as

$$
\operatorname{det}(\mathbf{F})=F_{33} \cdot\left(F_{11} F_{22}-F_{12} F_{21}\right) .
$$

According to Marek et al. [23], the determination of the component $F_{33}$ of the deformation gradient for each instant can be calculated as

$$
F_{33}(t)=1+\varepsilon_{33}(t)=1+\int_{0}^{t} \Delta \varepsilon_{33} \mathrm{~d} t,
$$

which leaves the increment of the out-of-plane strain component $\Delta \varepsilon_{33}$ to be calculated. In elasto-plasticity, assuming isotropic elastic behaviour, this component can be determined using Hooke's law and taking advantage of the isochoric character of plasticity as follows

$$
\Delta \varepsilon_{33}=-\frac{\nu}{1-\nu}\left(\Delta \varepsilon_{11}^{\mathrm{e}}+\Delta \varepsilon_{22}^{\mathrm{e}}\right)-\left(\Delta \varepsilon_{11}^{\mathrm{p}}+\Delta \varepsilon_{22}^{\mathrm{p}}\right),
$$

where $\Delta \varepsilon_{11}^{\mathrm{e}}$ and $\Delta \varepsilon_{22}^{\mathrm{e}}$ and $\Delta \varepsilon_{11}^{\mathrm{p}}$ and $\Delta \varepsilon_{22}^{\mathrm{p}}$ are the components of the elastic and plastic strain tensor, respectively, and $\nu$ is the Poisson's ratio.

The external virtual work is calculated based on the measured force along the test and constitutes a great asset for the VFM, because it is not required the distribution the force. Choosing a virtual field constant along the boundary $\Gamma_{0}^{f}$ (condition 2) simplifies the computation of the external virtual work as follows

$$
W_{\text {ext }}\left(\mathbf{U}^{*}\right)=\mathbf{U}^{*} \cdot \int_{\Gamma_{0}^{f}} \overline{\mathbf{T}} \mathrm{d} S=\mathbf{U}^{*} \cdot \mathbf{F}^{\text {load }} .
$$

Another important aspect in the calibration of non-linear constitutive models, such as elasto-plastic models, is the number of time instants taken into account to reconstruct the stress field. An accurate reconstruction of the stress field requires the use of several measures of the displacement field along the test. Therefore, Eq. 5 must be written as a sum of the principle of virtual work for each time instant. This is also a way to enrich the objective function. Finally, Eq. 5 can be written for different virtual fields in order to enrich the objective function and to explore the most relevant information from the reconstructed stress field. Hence, the objective function for the VFM can be written in a general form as

$$
\varphi(\boldsymbol{\xi})=\sum_{k=1}^{n_{\mathrm{v}}} \sum_{j=1}^{n_{\mathrm{t}}}\left(W_{\mathrm{int}}\left(\boldsymbol{\xi}, \mathbf{U}^{*(k)}, t_{j}\right)-W_{\mathrm{ext}}\left(\mathbf{U}^{*(k)}, t_{j}\right)\right)^{2},
$$

where $n_{\mathrm{v}}$ is the number of virtual fields selected and $n_{\mathrm{t}}$ the number of time steps considered. The identification of the material parameters is performed through the minimisation of Eq. 14. The minimum of the objective function is found when the identified material parameters in the selected constitutive model give rise to stress fields that minimise the difference between $W_{\text {int }}$ and $W_{\text {ext }}$ over the considered time instants. Generally, the reconstruction of the stress field for an elasto-plastic constitutive model requires an integration algorithm, in the same way as a finite element solver. There are several options for the integration algorithm, the classical one is based on the backward-Euler scheme combined with an elastic predictor/plastic corrector method to update the stress state. Examples of this kind of algorithm can be found in [34, 35]. Nevertheless, other algorithms can be used, for instance, the one proposed by Rossi et al. [22], called direct 


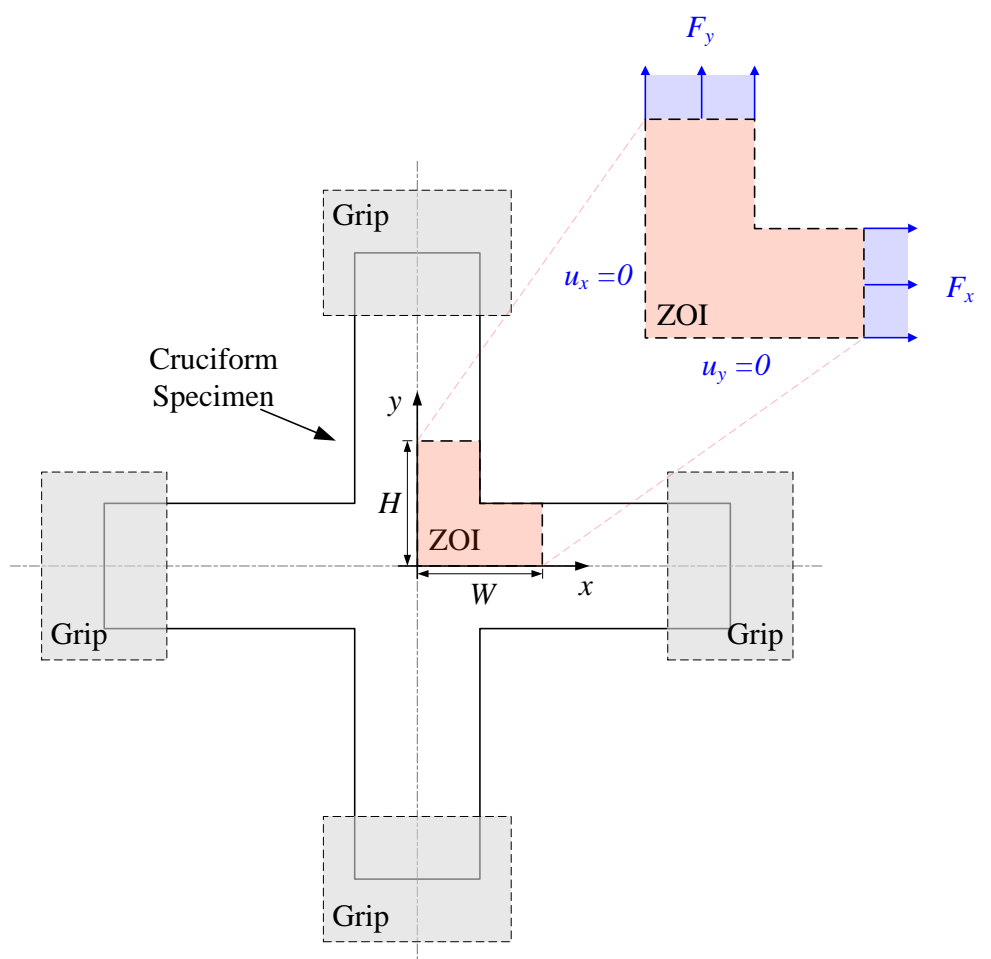

Figure 1: Illustrative example of a biaxial test with a cruciform specimen and respective zone of interest (ZOI) selected for the present study.

\subsection{Virtual fields definition and identification procedure}

Defining a set of virtual fields is one of the main tasks before using the VFM. In the framework of plasticity, three main strategies are currently available to select a suitable set of virtual fields [36]. The most commonly used strategy is manually defined virtual fields. In this strategy, the user has to develop a continuous function adapted to the problem in hands (that depends on the experimental geometry and boundary conditions), that generates the value of the virtual field over the considered domain. Usually, the functions are of polynomial or periodical basis. This approach has the disadvantage of relying on the experience of the user to develop the function. Nevertheless, this approach is easy to implement. The other two strategies were developed to overcome the disadvantage of the previous one, allowing to obtain the virtual field set automatically with some user inputs $[28,36]$. Nevertheless, these two automatic strategies have the disadvantage of being more difficult to implement than the manual strategy and require a higher computational effort. Therefore, in the present study the manual approach is chosen.

The set of virtual fields that will be presented can be used with any cruciform specimen. This set was developed to meet the above-mentioned conditions: to be null over the fixed boundaries (condition 1), where the prescribed displacement is zero, and constant over the boundaries at which the resultant of the applied force is known (condition 2). Consider the illustrative example of a general cruciform specimen under biaxial loading presented in Fig. 1. The set of developed virtual fields meets the above-mentioned conditions considering the zone of interest (ZOI) represented in Fig. 1, which corresponds to one-fourth of the central zone of the specimen. The boundaries with prescribed displacements $\left(u_{x}=u_{y}=0\right)$ are considered symmetry boundaries. For the boundaries marked with $F_{x}$ and $F_{y}$, the resultant of the applied force is considered to be known for the respective direction. 
The developed set of virtual field is the following:

$$
\begin{gathered}
\mathbf{U}^{*(1)}=\left\{\begin{array}{l}
U_{x}^{*}=\frac{X}{W} \\
U_{y}^{*}=0
\end{array},\right. \\
\mathbf{U}^{*(2)}=\left\{\begin{array}{l}
U_{x}^{*}=0 \\
U_{y}^{*}=\frac{Y}{H}
\end{array},\right. \\
\mathbf{U}^{*(3)}=U_{x}^{*}=U_{y}^{*}=\sin \left(\frac{X}{W} \pi\right) \sin \left(\frac{Y}{H} \pi\right) .
\end{gathered}
$$

The constants $W$ and $H$ represent the height and the width of the ZOI. $X$ and $Y$ represent the material coordinates in the global frame regarding the initial configuration. With this set of virtual fields the objective function presented in Eq. 14 is written for $n_{\mathrm{v}}=3$. The first two virtual fields Eq. 15 and Eq. 16 allow to write the external virtual work for the applied force in the $x$ - and $y$-directions, which makes possible to use the force applied in each arm of the specimen. The third virtual field activates all the components of the stress tensor in calculation of the internal work, since grad $\mathbf{U}^{*(3)}$ contains all the components active. Similar virtual fields can be found, for example, in [22, 37].

The objective function Eq. 14 is commonly normalised by the $W_{\text {ext }}$, but due to the selected virtual fields this is not possible, because the third virtual field Eq. 17 does not include the contribution of the $W_{\text {ext }}$. Therefore, Eq. 14 is normalised by the maximum $W_{\text {int }}$.

The theoretical background presented in this section, as well as the presented set of virtual fields, were implemented in an in-house code using the programming language Fortran. This code includes a gradientbased Levenberg-Marquardt optimisation method [38] used to minimise the objective function. The low computational cost and ease of implementation are the main assets of this gradient-based method. However, it is prone to reach local minima instead of a global one. In this study, the required Jacobian matrix for the Levenberg-Marquardt method is calculated by forward finite differences. The convergence criterion for this method was establish as: the objective function becomes lower than a tolerance or the relative difference between parameters in consecutive iterations is lower than or equal to $\epsilon=1 \times 10^{-10}$.

\section{Material model}

This section is dedicated to the elasto-plastic constitutive models used in this study. Two yield criteria are presented, Hill'48 and YLD2000-2d. In addition, as the present study is performed with virtual experimental data generated with two models which differ from each other in the adopted yield criterion, after presenting each yield criterion, the respective set of material parameters is also presented.

In this study, the elasto-plastic behaviour of sheet metals is modelled assuming additive decomposition of the strain rate tensor and associated flow rule [39]. It is considered isotropic linear elastic behaviour described by Hooke's law and anisotropic plastic behaviour. The remaining elements, the yield surface and the hardening law, are presented below. It should be mentioned that all equations are presented in the orthogonal anisotropic frame (or material frame), which axes coincide with rolling, transverse and normal directions of the sheet plane. Moreover, the anisotropy frame is assumed to have a constant angle regarding the co-rotational frame along the deformation process.

The yield surface in plasticity, assuming isotropic hardening, can be written as

$$
f=\bar{\sigma}(\boldsymbol{\sigma})-\sigma_{y}\left(\bar{\varepsilon}^{\mathrm{p}}\right)=0 .
$$

where $\bar{\sigma}(\boldsymbol{\sigma})$ is the equivalent stress defined by a yield criterion and $\sigma_{y}\left(\bar{\varepsilon}^{\mathrm{p}}\right)$ defines the flow stress. $\sigma_{y}\left(\bar{\varepsilon}^{\mathrm{p}}\right)$ is assumed as a function of a single internal variable, the equivalent plastic strain $\bar{\varepsilon}^{\mathrm{p}}$. Here, it is chosen the classical phenomenological Swift's law to describe the evolution of $\sigma_{y}\left(\bar{\varepsilon}^{\mathrm{p}}\right)$, which can be written as

$$
\sigma_{y}\left(\bar{\varepsilon}^{\mathrm{p}}\right)=K\left(\varepsilon_{0}+\bar{\varepsilon}^{\mathrm{p}}\right)^{n}, \quad \varepsilon_{0}=\left(\frac{\sigma_{0}}{K}\right)^{(1 / n)}
$$




\begin{tabular}{ccc|ccc}
\multicolumn{3}{c}{ Swift's law } & \multicolumn{3}{c}{ Hill'48 parameters } \\
\hline$\sigma_{0}[\mathrm{MPa}]$ & $n$ & $K[\mathrm{MPa}]$ & $F$ & $G$ & $N$ \\
160 & 0.26 & 565 & 0.2782 & 0.3731 & 1.5568 \\
\hline \multicolumn{3}{c}{$\sigma_{\alpha}$} & \multicolumn{3}{c}{$r_{\alpha}$} \\
\hline$\sigma_{0}$ & $\sigma_{45}$ & $\sigma_{90}$ & $r_{0}$ & $r_{45}$ & $r_{90}$ \\
1.000 & 1.030 & 1.051 & 1.680 & 1.890 & 2.253 \\
\hline
\end{tabular}

Table 1: Parameters for Swift's law and Hill'48 yield, and normalized yield stress values $\left(\sigma_{\alpha}\right)$ and plastic anisotropy coefficients $\left(r_{\alpha}\right)$ for $0^{\circ}, 45^{\circ}$ and $90^{\circ}$ according to the axis angle $(\alpha)$ between the rolling and tensile directions.

$\sigma_{0}, K$ and $n$ are material parameters which must be identified according to the material. $\sigma_{0}$ is the initial yield stress and is assumed as a material parameter instead of $\varepsilon_{0}$, because when $\varepsilon_{0}$ is taken as the material parameter to be identified, the errors are usually high, due to the small order of magnitude of this parameter, but without significant impact on the initial yield stress $[14,40]$.

The first anisotropic yield criterion adopted is the quadratic criterion proposed by Hill [9], which is commonly called Hill'48 yield criterion. Assuming plane stress condition, this criterion has the following form

$$
\bar{\sigma}^{2}=H\left(\sigma_{x x}-\sigma_{y y}\right)^{2}+G \sigma_{x x}^{2}+F \sigma_{y y}^{2}+2 N \sigma_{x y}^{2}
$$

$H, G, F$ and $N$ are the material parameters. $\sigma_{x x}, \sigma_{y y}$ and $\sigma_{x y}$ are the components of the Cauchy stress tensor in the anisotropic frame. It is often assumed, for matters of convenience, that the yield stress in the rolling direction corresponds to $\sigma_{y}$, leading to the condition $G+H=1$. Following a direct procedure, the parameters $G, F$ and $N$ can be identified with closed-form solutions either as a function of the yield stresses or the plastic anisotropy coefficients. However, each approach gives different results [11]. The most common approach makes use of the plastic anisotropy coefficients $\left(r_{\alpha}\right)$ in the directions $0^{\circ}, 45^{\circ}$ and $90^{\circ}$ from the rolling direction. In this case the $G, F$ and $N$ are determined as

$$
G=\frac{1}{1+r_{0}} ; \quad F=\frac{r_{0}}{r_{90}\left(1+r_{0}\right)} ; \quad N=\frac{\left(r_{0}+r_{90}\right)\left(2 r_{45}+1\right)}{2 r_{90}\left(1+r_{0}\right)} .
$$

In this study, Hill'48 yield criterion is combined with Swift's law to describe the behaviour of a typical mild steel. The reference material parameters associated to this model are presented in Table 1 . The values of the elastic parameters are: Young's modulus $E=210 \mathrm{GPa}$ and Poisson's ratio $\nu=0.3$. Moreover, the normalised yield stress values $\left(\sigma_{\alpha}\right)$ and the plastic anisotropy coefficients $\left(r_{\alpha}\right)$ for different angles between the tensile and rolling direction $\left(\alpha=0^{\circ}, 45^{\circ}\right.$ and $\left.90^{\circ}\right)$ are also presented in Table 1.

The second yield criterion adopted in this study is non-quadratic and was proposed by Barlat et al. [12]. Named YLD2000-2d, this yield criterion was formulated for plane stress conditions and can be expressed as

$$
2 \bar{\sigma}^{a}=\left|X_{1}^{\prime}-X_{2}^{\prime}\right|^{a}+\left|2 X_{2}^{\prime \prime}+X_{1}^{\prime \prime}\right|^{a}+\left|2 X_{1}^{\prime \prime}+X_{2}^{\prime \prime}\right|^{a},
$$

where $a$ is a material parameter that usually assumes the value of 6 or 8 , depending on the crystallographic structure of the material. $X_{1}^{\prime}, X_{2}^{\prime}$ and $X_{1}^{\prime \prime}, X_{2}^{\prime \prime}$ are the principal values of the tensors $\mathbf{X}^{\prime}$ and $\mathbf{X}^{\prime \prime}$ obtained after two linear transformations on the deviatoric stress tensor. In a simple way, these can be determined directly from the Cauchy stress tensor as

$$
\mathbf{X}^{\prime}=\mathbf{L}^{\prime} \boldsymbol{\sigma} ; \quad \mathbf{X}^{\prime \prime}=\mathbf{L}^{\prime \prime} \boldsymbol{\sigma},
$$




\begin{tabular}{ccc|ccc|ccc}
\multicolumn{3}{c}{ Swift's law } & \multicolumn{3}{c}{$\sigma_{\alpha}$} \\
\hline$\sigma_{0}[\mathrm{MPa}]$ & $n$ & $K[\mathrm{MPa}]$ & $\sigma_{0}$ & $\sigma_{45}$ & $\sigma_{90}$ & $r_{0}$ & $r_{45}$ & $r_{90}$ \\
212.03 & 0.239 & 385.47 & 1.0 & 0.984 & 0.944 & 0.94 & 0.39 & 0.64 \\
\hline \multicolumn{1}{c}{ YLD2000-2d parameters } \\
\hline$\alpha_{1}$ & $\alpha_{2}$ & $\alpha_{3}$ & $\alpha_{4}$ & $\alpha_{5}$ & $\alpha_{6}$ & $\alpha_{7}$ & $\alpha_{8}$ & $m$ \\
0.9580 & 1.045 & 0.9485 & 1.0568 & 0.9938 & 0.9397 & 0.9200 & 1.1482 & 8.0 \\
\hline
\end{tabular}

Table 2: Parameters for Swift's law and YLD2000-2d yield criterion and normalized yield stress values $\left(\sigma_{\alpha}\right)$ and plastic anisotropy coefficients $\left(r_{\alpha}\right)$ for $0^{\circ}, 45^{\circ}$ and $90^{\circ}$ according to the axis angle $(\alpha)$ between the rolling and tensile directions.

where $\mathbf{L}^{\prime}$ and $\mathbf{L}^{\prime \prime}$ can be defined based on eight parameters as

$$
\begin{aligned}
& \left\{\begin{array}{l}
L_{11}^{\prime} \\
L_{12}^{\prime} \\
L_{21}^{\prime} \\
L_{22}^{\prime} \\
L_{66}^{\prime}
\end{array}\right\}=\left[\begin{array}{ccc}
2 / 3 & 0 & 0 \\
-1 / 3 & 0 & 0 \\
0 & -1 / 3 & 0 \\
0 & 2 / 3 & 0 \\
0 & 0 & 1
\end{array}\right]\left\{\begin{array}{l}
\alpha_{1} \\
\alpha_{2} \\
\alpha_{7}
\end{array}\right\} \text { and } \\
& \left\{\begin{array}{l}
L_{11}^{\prime \prime} \\
L_{12}^{\prime \prime} \\
L_{21}^{\prime \prime} \\
L_{22}^{\prime \prime} \\
L_{66}^{\prime \prime}
\end{array}\right\}=\frac{1}{9}\left[\begin{array}{ccccc}
-2 & 2 & 8 & -2 & 0 \\
1 & -4 & -4 & 4 & 0 \\
4 & -4 & -4 & 1 & 0 \\
-2 & 8 & 2 & -2 & 0 \\
0 & 0 & 0 & 0 & 9
\end{array}\right]\left\{\begin{array}{l}
\alpha_{3} \\
\alpha_{4} \\
\alpha_{5} \\
\alpha_{6} \\
\alpha_{8}
\end{array}\right\}
\end{aligned}
$$

where $\alpha_{k}(k=1, \ldots, 8)$ are the parameters to be identified. The identification of the $\alpha_{k}$ parameters is conventionally performed with the method proposed by Barlat et al. [12] which requires the yield stresses $\sigma_{0}, \sigma_{45}$ and $\sigma_{90}$ and the plastic anisotropic coefficients $r_{0}, r_{45}$ and $r_{90}$. Moreover, it also requires the biaxial yield stress $\sigma_{b}$ and the biaxial anisotropy coefficient $r_{b}$. Using this data, $\alpha_{k}(k=1, \ldots, 8)$ can be identified using an optimisation method, such as the Newton-Raphson method as suggested by Barlat et al. [12].

Similar to Hill'48, assuming $\sigma_{y}$ as the yield stress in the rolling direction leads to the following condition

$$
\left\{\frac{1}{2}\left[\left|\frac{2 \alpha_{1}+\alpha_{2}}{3}\right|^{a}+\left|\frac{2\left(\alpha_{3}-\alpha_{4}\right)}{3}\right|^{a}+\left|\frac{4 \alpha_{5}-\alpha_{6}}{3}\right|^{a}\right]\right\}^{(1 / a)}=1 .
$$

This condition is not usually imposed, to the best of the authors' knowledge, except in [41].

The YLD2000-2d is combined with Swift's law to model the behaviour of an aluminium alloy AA6016 in T4 state [42]. The material parameters for this model are presented in Table 2. The elastic parameters are: $E=70 \mathrm{GPa}$ and $\nu=0.33$. Moreover, the normalised yield stress values and the plastic anisotropy coefficients are also presented [42].

\section{Cruciform geometries}

In this section, three cruciform geometries used in biaxial tension are presented as well as the respective numerical models. Stress and strain fields are analysed for each geometry. In order to simplify the notation, the three geometries used in this work are called Cr1, Cr2 and Cr3. The first geometry selected was proposed 


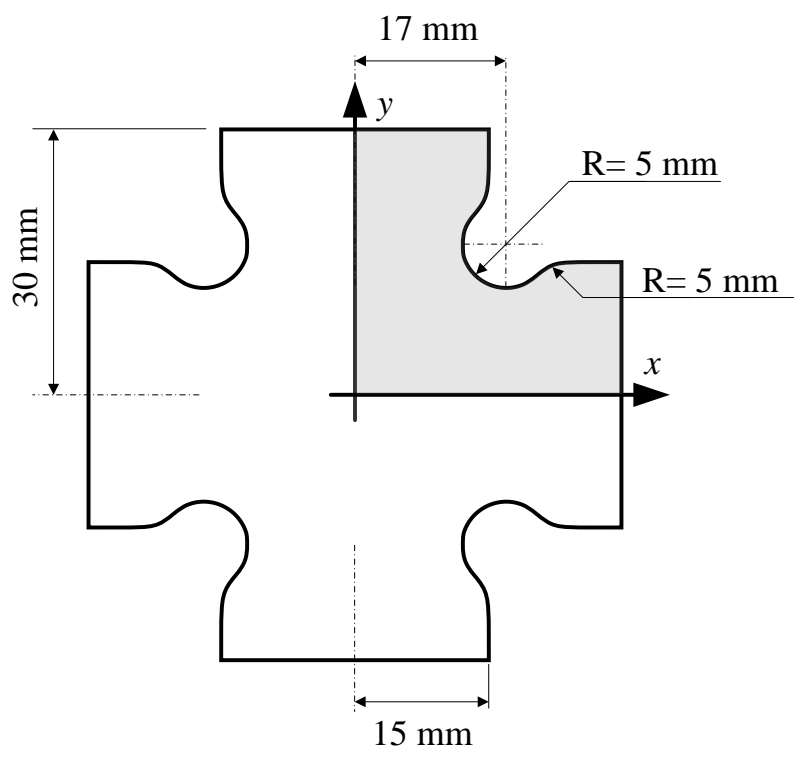

(a) $\mathrm{Cr} 1$

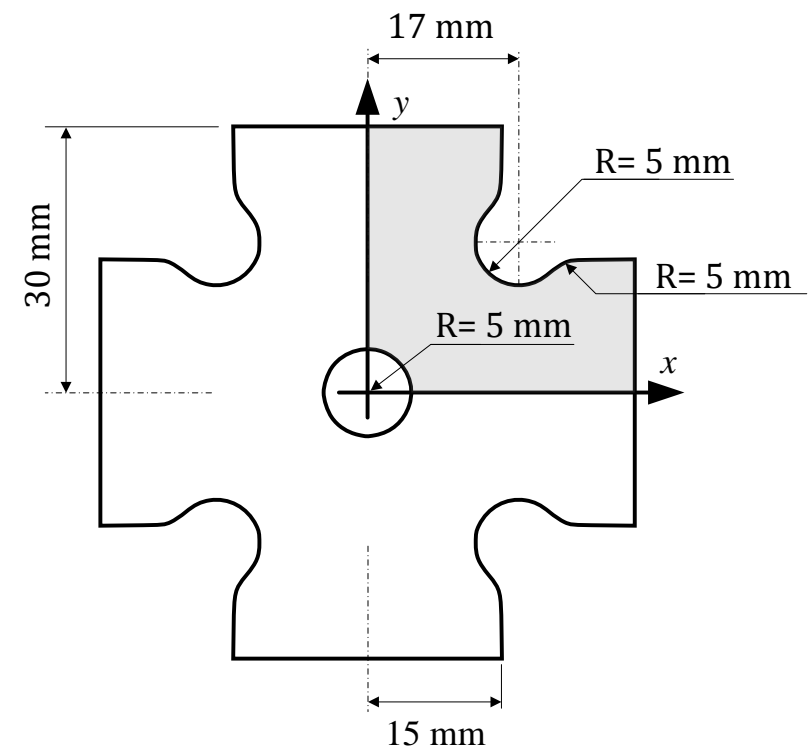

(b) $\mathrm{Cr} 2$

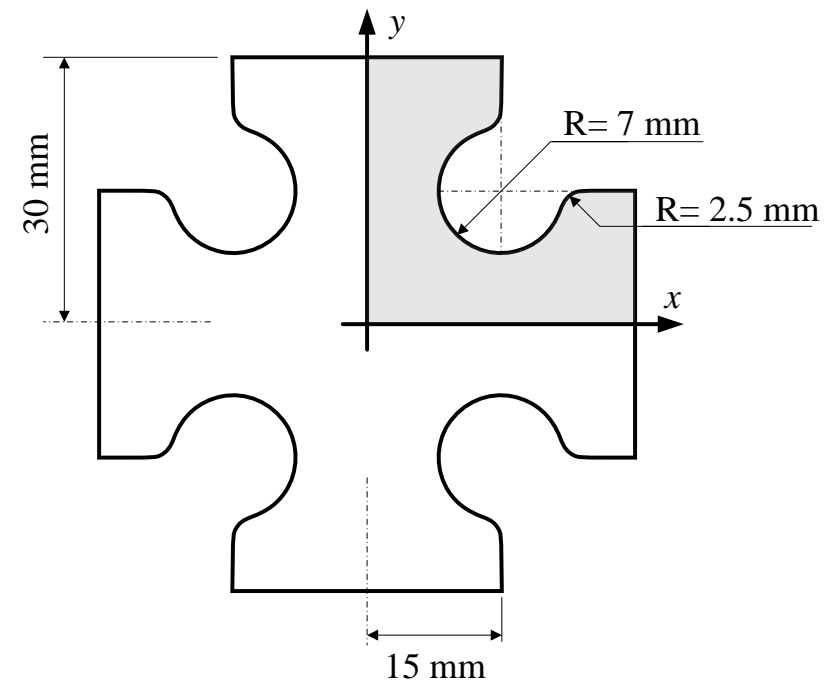

(c) $\mathrm{Cr} 3$

Figure 2: Cruciform geometries: (a) Cr1 has been proposed by Zhang et al. [16], (b) $\mathrm{Cr} 2$ and (c) Cr3 are adaptations of Cr1. The grey areas represent the geometry of the FE model. 
by Zhang et al. [16] to identify the material parameters of Bron and Besson yield criterion [18]. The results of this study showed that the proposed geometry gives enough information for an accurate identification of a yield criterion. The dimensions and the geometry of this specimen are presented in Fig. 2a. The other two geometries are adaptations of Cr1. The second geometry (Fig. 2b) has the same dimensions as Cr1, but contains a hole in the centre. The hole has the particular role of increasing the strain field heterogeneity and thus, increasing the sensitivity of the strain field to the material parameters. The inclusion of a hole in the geometry with this aim was reported in several studies such as, [20, 43, 44, 45]. For the third specimen (Fig. 2c), the rounding radius at the intersection of the arms has been changed.

The three geometries are numerically tested in the same conditions. Due to the material and geometrical symmetries, the FE models represent one fourth of the respective specimen geometry (grey areas in Fig. 2). Moreover, plane stress conditions and constant thickness of the sheet are assumed. The simulation is displacement-driven, with a displacement of $2 \mathrm{~mm}$ applied to each arm of the specimen and symmetry boundary conditions. ABAQUS standard software is used to perform the numerical analysis. The element CPS4 (bilinear shape functions, full integration) is used, as well as a large strain formulation. The mesh density for the three geometries is selected based on a convergence study. High mesh densities have been tested without significant effect on the strain and stress distributions. Therefore, the chosen mesh density has an average size of the elements of $0.5 \mathrm{~mm}$, which results in a total number of elements: 2480,2089 and 2102 for $\mathrm{Cr} 1, \mathrm{Cr} 2$ and $\mathrm{Cr} 3$, respectively. Indeed, similar mesh densities have been used in different works with cruciform specimens, for example [5, 16, 44].

The simulations are run with adaptive time stepping. The model composed by Swift's law and Hill'48 yield criterion is used, the material parameters for this constitutive model are presented in Table 1. For all the geometries it is assumed that rolling direction coincides with the $x$-axis represented in Fig. 2.

The equivalent plastic strain distribution at the last increment and the deformed FE meshes for the three geometries are presented in Fig. 3. Cr1 (Fig. 3a) contains the largest quasi-homogeneous area in the centre of the specimen, even though with lower values of equivalent plastic strain, which is a common characteristic (The highest values of the equivalent plastic strain are located in the arms and at the rounding radius at the intersection of the two arms, with a maximum value of $\bar{\varepsilon}^{\mathrm{p}}=0.31$. Cr2 (Fig. 3b) loses completely the homogeneous area in the centre and has the highest concentration of equivalent plastic strain in the diagonal direction near the rounding radius at the intersection of the two arms and in the vicinity of the hole. The highest value is $\bar{\varepsilon}^{\mathrm{p}}=0.29$, slightly lower than for Cr1. In the case of Cr3 (Fig. 3c),

235 a quasi-homogeneous area develops in the centre of the specimen, still with a smaller area than Cr1. The maximum value of equivalent plastic strain is lower in this case with $\bar{\varepsilon}^{\mathrm{p}}=0.24$. The range of plastic strain obtained in a specimen generally influences the identification results, specifically the identification of the hardening law.

The diversity of strain or stress states is also an important factor for an accurate calibration of a constitutive model, specially when a complex yield criterion has to be calibrated $[17,43,37]$. Therefore, the plot of the strain and stress states in the principal axes (in-plane) for the three geometries is presented in Fig. 4. $\varepsilon_{1}$ and $\varepsilon_{2}$ represent the major and minor strains, and $\sigma_{1}$ and $\sigma_{2}$ the major and minor stresses. It should be noted that in the three cases the whole specimen's surface is considered for these plots and for the remaining analysis. In the three cases, the stress states close to tension are predominant and the first 245 quadrant in the principal stress space $\left(\sigma_{1}>0\right.$ and $\left.\sigma_{2}>0\right)$ is the most populated one. Nevertheless, Cr3 (see Fig. 4 (e-f)) shows the widest distribution of strain and stress states. It is possible to see an increase of points in the fourth quadrant in the principal stress space $\left(\sigma_{1}>0\right.$ and $\left.\sigma_{2}<0\right)$ compared with the other two geometries, which gives weight to shear components in the identification procedure. In terms of spatial distribution, the region of equi-biaxial tension is located in the specimen centre, in the case of $\mathrm{Cr} 1$ and $\mathrm{Cr} 3$, whereas for $\mathrm{Cr} 2$, this region is divided in two and can be found near the vicinity of the hole. Along the arms of the specimens Cr1 and Cr2, the stress state evolves from equi-biaxial to tension, whereas for Cr3, stress states between simple shear and tension are also observed. A common feature of the three geometries is the equi-biaxial tension state represented by low values of strain, which is in accordance with the literature. Moreover, Fig. 5 shows the rotation angle $(\theta)$ between principal stress base and material frame for Cr3. This measure can be used to highlight the sensitivity of the test to anisotropy. It can be seen that the cruciform specimen exhibits a rather wide distribution of the rotation angle. In particular, in the arms, where the 


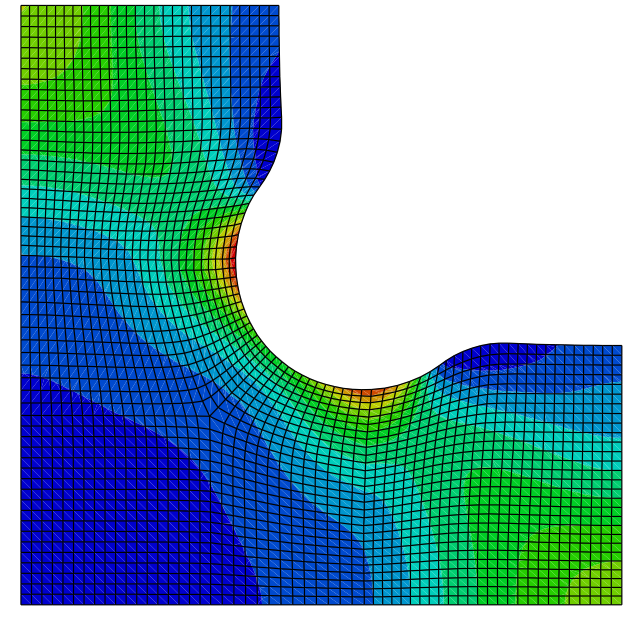

(a) $\mathrm{Cr} 1$

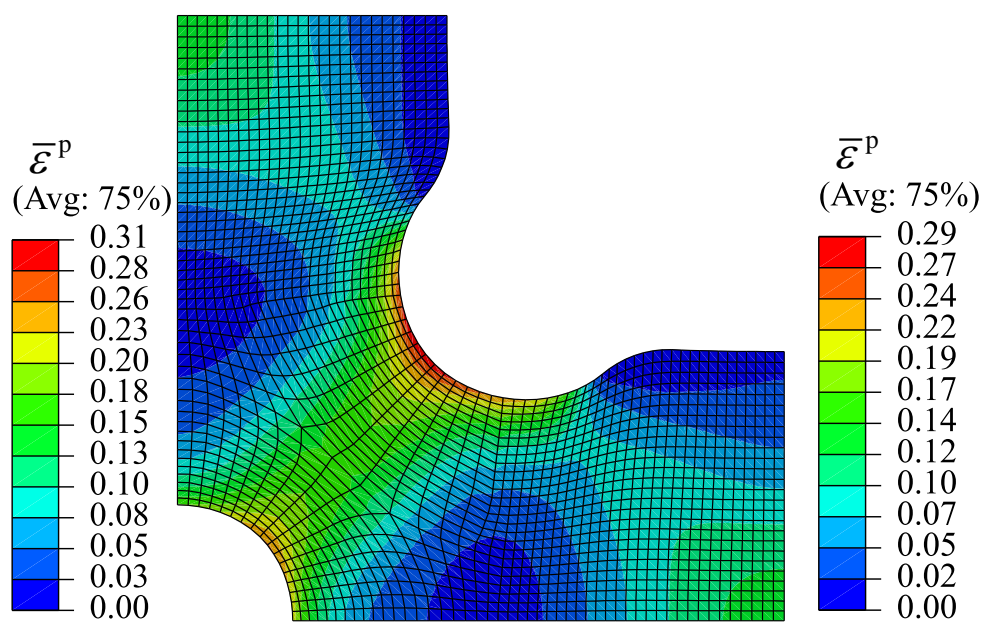

(b) $\mathrm{Cr} 2$

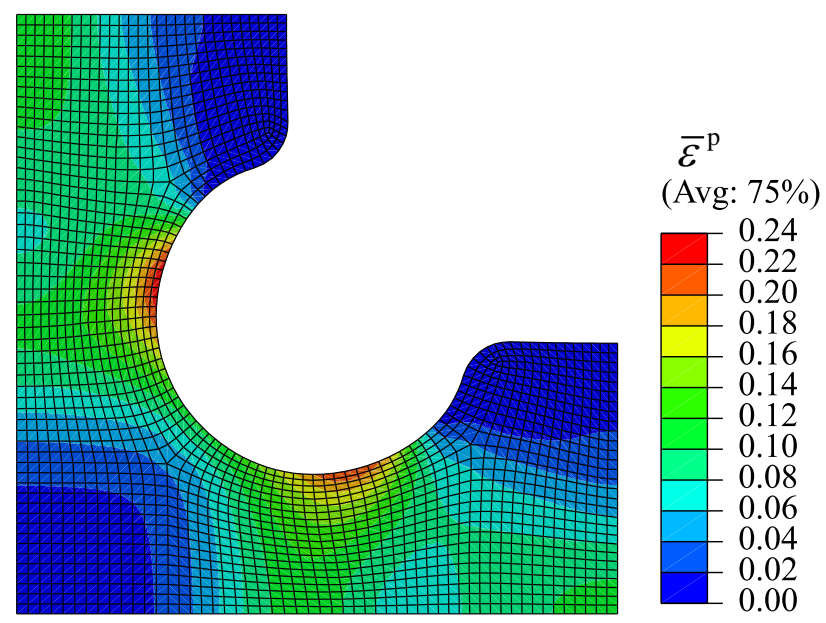

(c) $\mathrm{Cr} 3$

Figure 3: Equivalent plastic strain distribution for the last increment and deformed FE mesh for the three geometries. 
equivalent plastic strain is the highest, values close to $0^{\circ}$ and $90^{\circ}$ are well represented, as well as two areas with values close to $45^{\circ}$. Such a distribution highlights the richness of the test for the prediction of the parameters related to anisotropy. Note that similar distributions of the rotation angle are obtained for the two other geometries.

The evolution of the force applied to arms of the cruciform specimen is also presented in Fig. 6 for the three geometries. Due to the anisotropic behaviour of the material, there is a difference on the force applied to the horizontal and vertical arms. For the three geometries the force applied in the $y$-direction is higher than in the $x$-direction. 


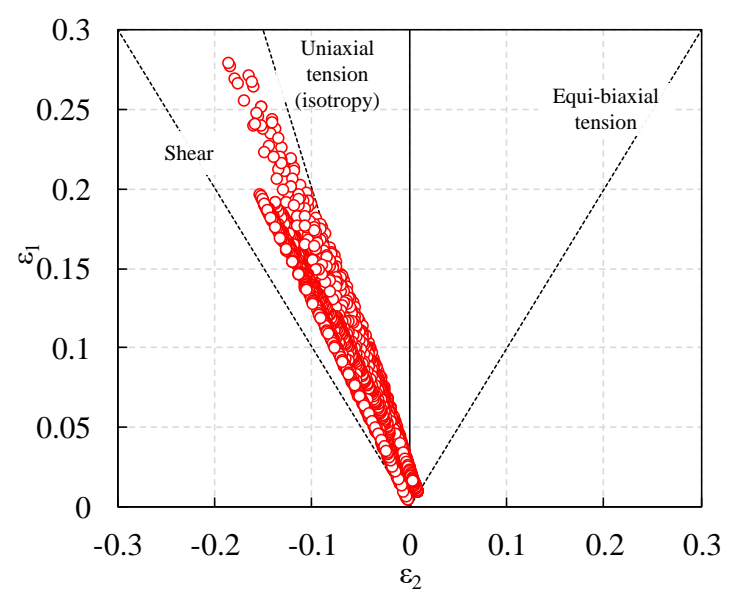

(a)

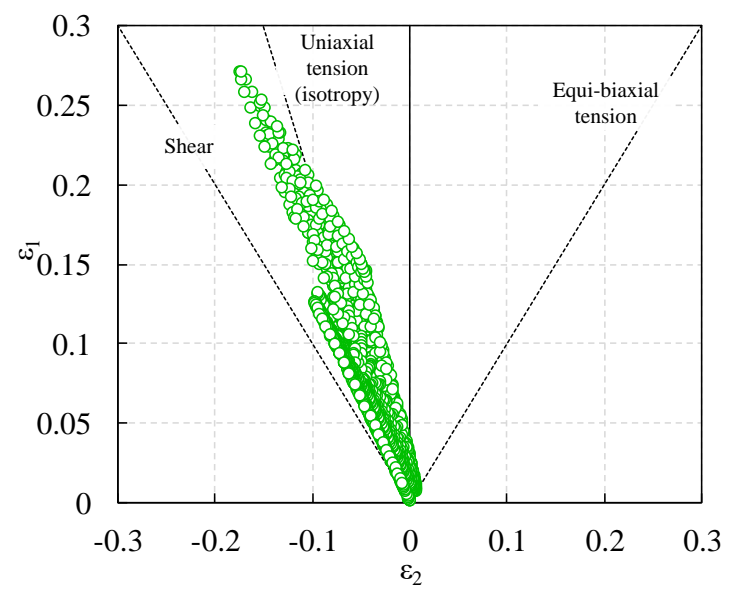

(c)

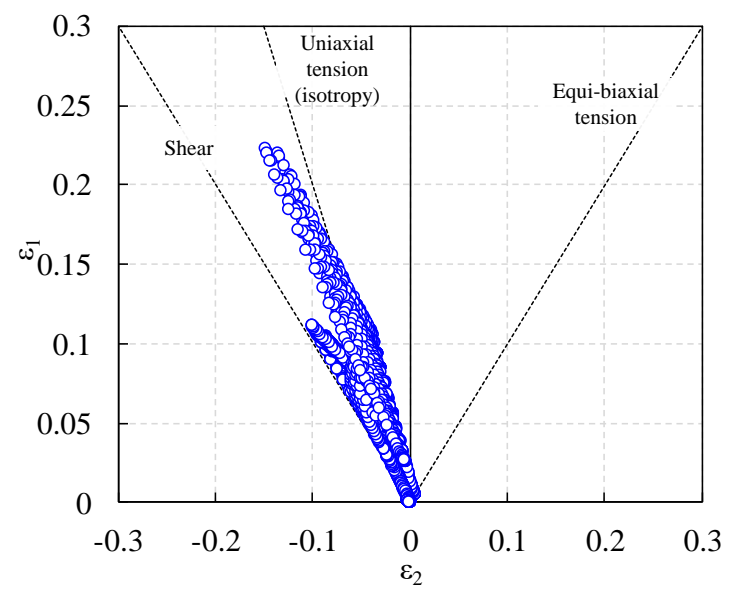

(e)

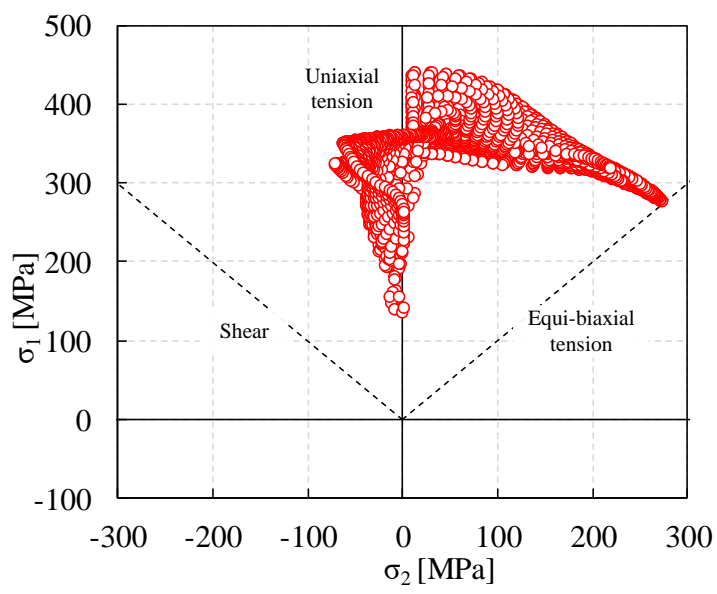

(b)

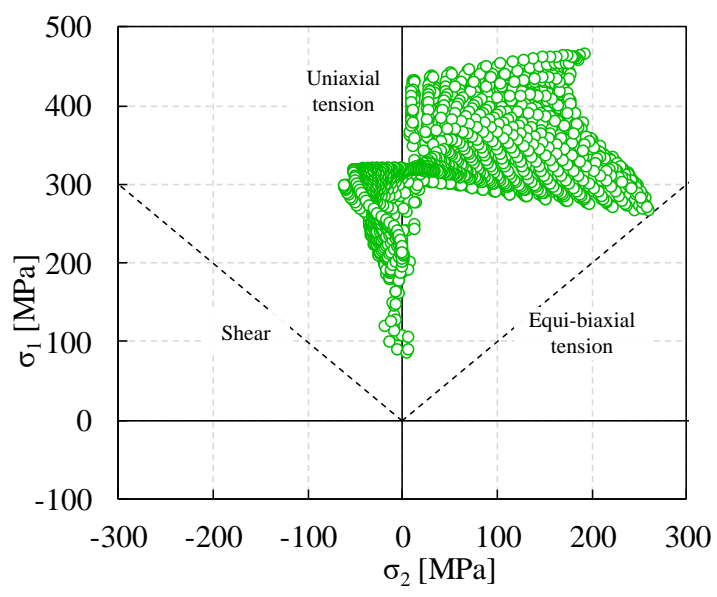

(d)

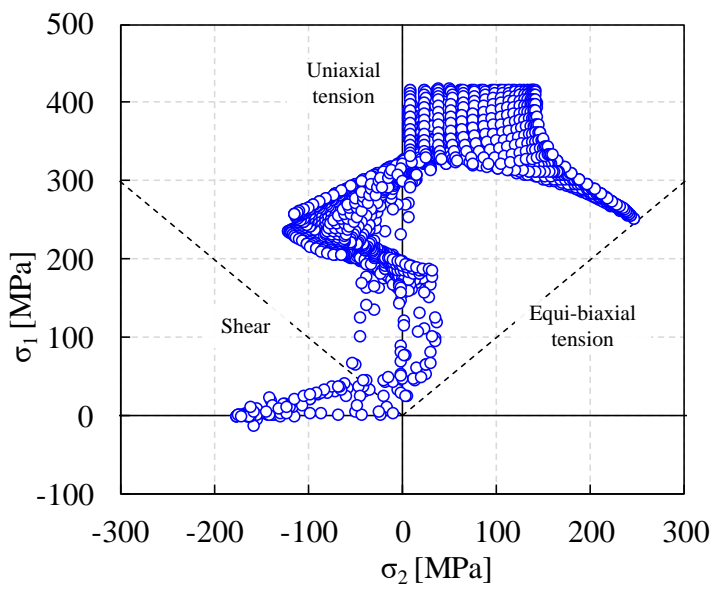

(f)

Figure 4: Plot of the principal strain and stress fields at the end of the tests: (a) and (b) Cr1, (c) and (d) Cr2, and (c) and (d) Cr3. 


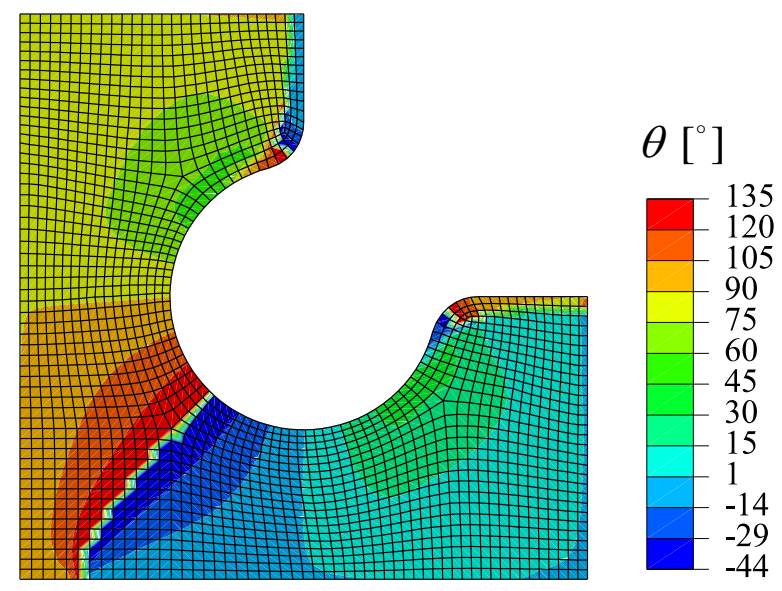

Figure 5: Rotation angle between principal stress base and material frame for Cr3.

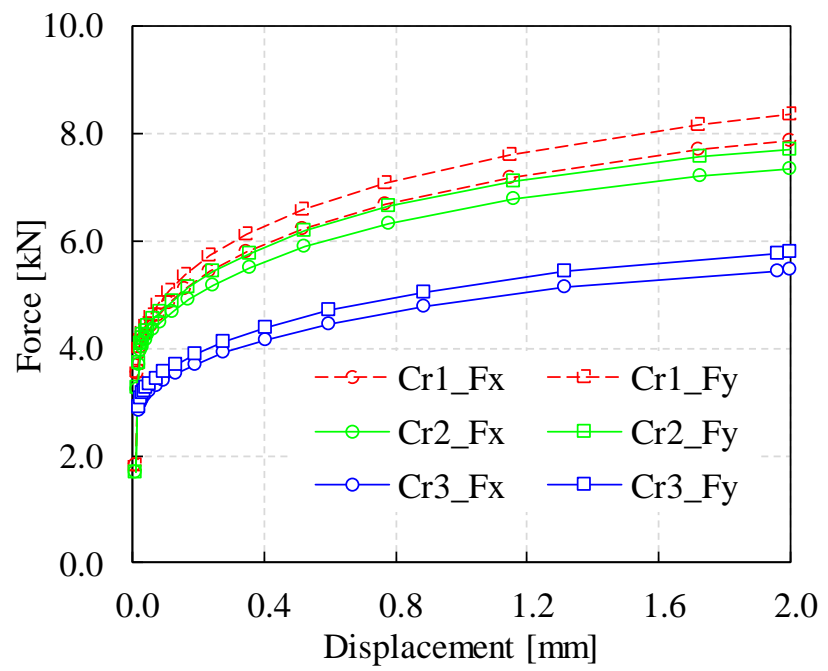

Figure 6: Force vs displacement for the three geometries: $\mathrm{Cr} 1, \mathrm{Cr} 2$ and $\mathrm{Cr} 3$. 


\section{Results}

\subsection{Hill'48 identification}

In this first subsection, the three geometries presented in section 4 are evaluated. The FE meshes presented in Fig. 3 are used with the VFM for the identification procedure. The model composed by Hill'48 yield criterion and Swift's law is selected to be calibrated. This model contains a total of 6 material parameters that are identified simultaneously using the displacement fields obtained for each cruciform geometry with the material parameters of Table 1. The accurate calibration of such a model can be hampered by the presence of local minima in the objective function, as reported by Kim et al. [37]. Hence, to check the presence of local minima, two initial sets of parameters are tested to initiate the optimisation problem. For the sake of simplicity they are called Sup_set and Inf_set and are presented in Table 3. The two sets differ on the values of the hardening parameters, whereas the material parameters of Hill' 48 yield criterion always start from the isotropic case. The two sets of hardening parameters represent flow curves above and below the reference one. The number of time steps $n_{\mathrm{t}}$ considered is the same required by ABAQUS to solve the FE simulation, which corresponds to 17, 19, and 17, for Cr1, Cr2 and Cr3, respectively. The distribution of the loading steps along the evolution of the applied displacement is represented in Fig. 6 by squares and circles. Note that the distribution of time steps is non-uniform. Nevertheless, it was shown previously that the VFM is not sensitive to the distribution of the time steps along the test, in contrast to the FEMU strategy [46]. Furthermore, the integration of the constitutive model for the reconstruction of the stress field in the VFM is performed using a backward-Euler algorithm, which was also implemented in a UMAT subroutine for ABAQUS standard.

The results of the identification process are presented in Table 4, as well as the absolute error calculated for each parameter. Regardless of the errors achieved, the identification process proved to be robust in finding a global minimum. Indeed, for each geometry, the two initial sets of parameters reach identical solutions.

The hardening parameters $\left(\sigma_{0}, n\right.$ and $\left.K\right)$ are accurately retrieved regardless of the geometry selected. Despite the fact that the three geometries reach different levels of equivalent plastic strain, there is no influence on the hardening behaviour identification, considering that the absolute error for $\sigma_{0}, n$ and $K$ is always lower than $1 \%$. In certain cases, the range of equivalent plastic strain attained can be important for the identification process [47]. Fig. 7 presents a comparison between the evolution of the applied force, shown in Fig. 6, and the computed force calculated on the basis of the internal virtual work and the material parameters identified. In this case, the solutions obtained with the Inf_set are used. The force can be calculate from the internal virtual work, since at the end of the optimisation, $W_{\text {int }} \approx W_{\text {ext }}$; combining this with Eq. 13, gives $W_{\text {int }} \approx W_{\text {ext }} \approx \mathbf{U}^{*} \cdot \mathbf{F}^{\text {load }}[22]$. Considering the definition of the virtual fields 1 and 2 , Eq. 15 and Eq. 16, $W_{\text {int }} \approx F^{\text {load }}$. For the three geometries, there is a clear agreement between this load calculation and FE predictions, which means the identified material parameters are able to describe the force in $x$ and $y$ directions.

Nevertheless, the highest absolute errors come from the yield criterion parameters, particularly from the parameter $N$ which is strictly related to the in-plane shear component $\sigma_{x y}$. The identifications performed with the data from $\mathrm{Cr} 1$ and $\mathrm{Cr} 2$ have the largest errors for the parameter $N$, above $25 \%$ and $5 \%$, respectively. Since the same identification procedure is used for the three geometries, the only thing that makes the

\begin{tabular}{ccccccc}
\cline { 2 - 7 } & $\sigma_{0}[\mathrm{MPa}]$ & $n$ & $K[\mathrm{MPa}]$ & $F$ & $G$ & $N$ \\
\hline Reference & 160 & 0.26 & 565 & 0.2782 & 0.3731 & 1.5568 \\
\hline Sup_set & 234 & 0.35 & 965 & 0.5 & 0.5 & 1.5 \\
Inf_set & 100 & 0.08 & 165 & 0.5 & 0.5 & 1.5 \\
\hline
\end{tabular}

Table 3: Reference parameters and two initial sets of parameters. 
Table 4: Comparison of the three cruciform geometries (Fig. 2) in the calibration of Swift's law and Hill'48 yield criterion. The results are presented for the two different initial sets of parameters Sup_set and Inf_set.

\begin{tabular}{|c|c|c|c|c|c|c|c|}
\hline & \multirow{2}{*}{\multicolumn{2}{|c|}{$\sigma_{0}[\mathrm{MPa}]$}} & & & & & \\
\hline & & & $n$ & $K[\mathrm{MPa}]$ & $F$ & $G$ & $N$ \\
\hline \multirow{5}{*}{ Cr1 } & Reference & 160 & 0.26 & 565 & 0.2782 & 0.3731 & 1.5568 \\
\hline & Sup_set & 160.35 & 0.262 & 566.11 & 0.2807 & 0.3758 & 1.9504 \\
\hline & Error & $0.22 \%$ & $0.85 \%$ & $0.20 \%$ & $0.87 \%$ & $0.72 \%$ & $25.29 \%$ \\
\hline & Inf_set & 160.38 & 0.263 & 566.40 & 0.2807 & 0.3758 & 1.9662 \\
\hline & Error & $0.23 \%$ & $0.99 \%$ & $0.25 \%$ & $0.88 \%$ & $0.70 \%$ & $26.30 \%$ \\
\hline \multirow{4}{*}{$\mathrm{Cr} 2$} & Sup_set & 160.66 & 0.258 & 566.07 & 0.2842 & 0.3786 & 1.6455 \\
\hline & Error & $0.41 \%$ & $0.76 \%$ & $0.19 \%$ & $2.15 \%$ & $1.46 \%$ & $5.70 \%$ \\
\hline & Inf_set & 160.51 & 0.258 & 565.62 & 0.2835 & 0.3779 & 1.6302 \\
\hline & Error & $0.32 \%$ & $0.67 \%$ & $0.11 \%$ & $1.89 \%$ & $1.27 \%$ & $4.72 \%$ \\
\hline \multirow{4}{*}{ Cr3 } & Sup_set & 159.76 & 0.261 & 566.40 & 0.2788 & 0.3735 & 1.5753 \\
\hline & Error & $0.15 \%$ & $0.44 \%$ & $0.25 \%$ & $0.22 \%$ & $0.10 \%$ & $1.19 \%$ \\
\hline & Inf_set & 159.74 & 0.261 & 566.16 & 0.2788 & 0.3735 & 1.5707 \\
\hline & Error & $0.16 \%$ & $0.37 \%$ & $0.21 \%$ & $0.21 \%$ & $0.09 \%$ & $0.89 \%$ \\
\hline
\end{tabular}

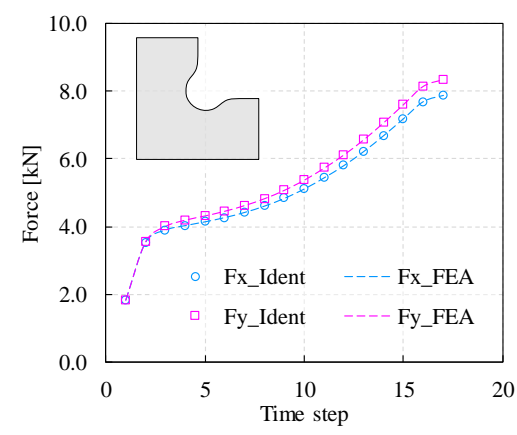

(a) $\mathrm{Cr} 1$

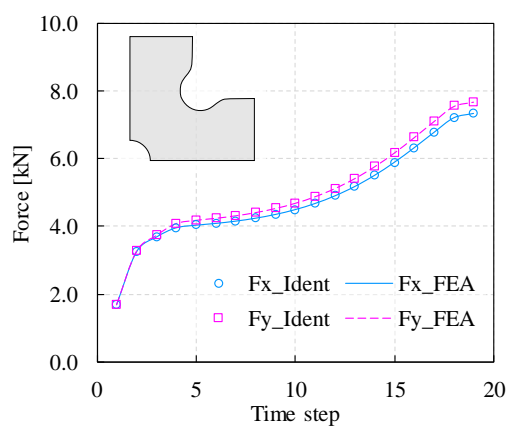

(b) $\mathrm{Cr} 2$

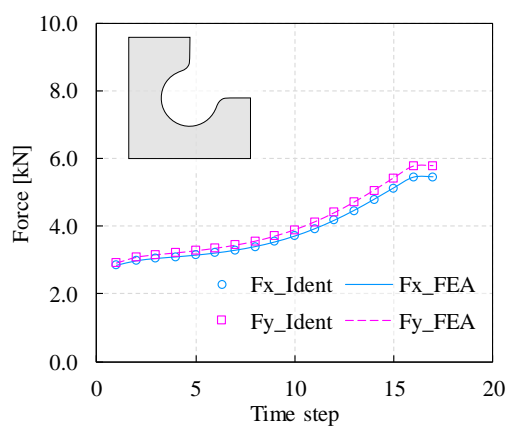

(c) Cr3

Figure 7: Comparison between computed force from the identified parameters (Fx_Ident and Fy_Ident) and the resultant force from the FE analysis for the three geometries (Fx_FEA and Fy_FEA) for $x$ and $y$-directions. 


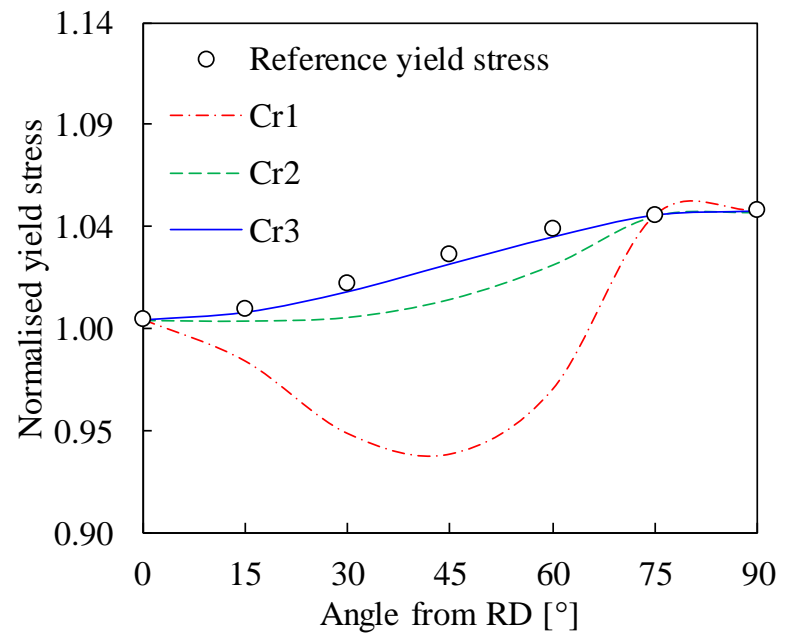

(a)

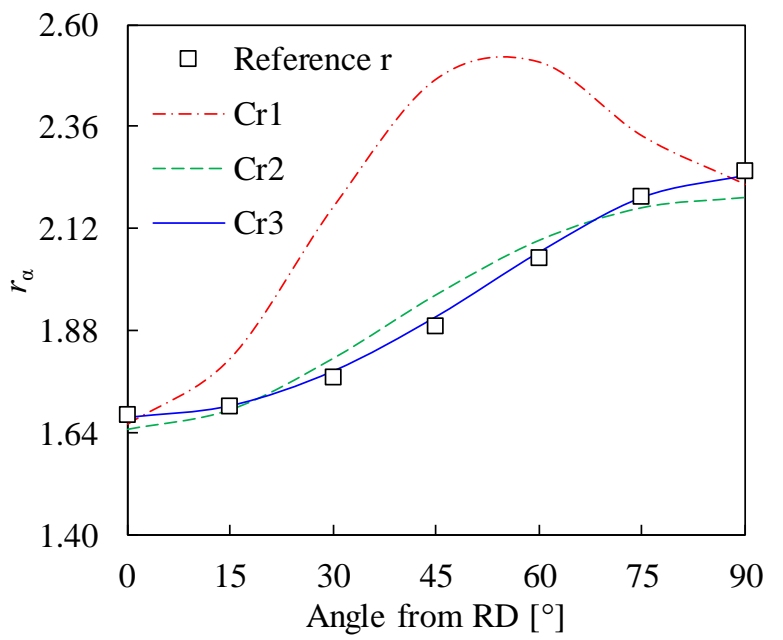

(b)

Figure 8: Evolution of the (a) normalised yield stress and (b) plastic anisotropy coefficient according to the tension axis angle from the rolling direction for the identified parameters with Cr1, Cr2 and Cr3.

possible to see that $\mathrm{Cr} 2$ has a wider dispersion of strain and stress states than Cr1. Consequently, the error is reduced from $25 \%$ to $5 \%$ for the parameter $N$. Cr3 gives the lowest error for the parameter $N$, around $1 \%$, which is a reasonable result. This can be explained by the fact that Cr3 has the widest dispersion in the fourth quadrant $\left(\sigma_{1}>0\right.$ and $\left.\sigma_{2}<0\right)$ of the principal stress space (see Fig. 4f), which gives a higher weight to the shear component and, consequently, improves the identification of the parameter $N$.

In order to verify the validity of the identified yield criterion parameters presented in table 4 , the evolution of the normalised yield stress and plastic anisotropy coefficient $\left(r_{\alpha}\right)$ value as a function of $\alpha$ is assessed in Fig. 8. The results for each geometry are obtained with the solution corresponding to the parameter set Inf_set. Both the normalised yield stresses and plastic anisotropy coefficients are well described with the identified parameters from Cr3. However, it is possible to observe a slight underestimation of the normalised yield stress for the angles $30^{\circ}, 45^{\circ}$ and $60^{\circ}$, which is a consequence of the error in the parameter $N$. The same tendency is observed for the other two sets of parameters obtained with $\mathrm{Cr} 1$ and $\mathrm{Cr} 2$. In the case of the plastic anisotropy coefficient, the parameters obtained with the $\mathrm{Cr} 1$ and $\mathrm{Cr} 2$ overestimate this coefficient for the angles $30^{\circ}, 45^{\circ}$ and $60^{\circ}$, but tend to give better estimations near the angles $0^{\circ}$ and $90^{\circ}$.

Since the results of $\mathrm{Cr} 3$ are the most accurate, the remaining analysis is focused on these results. Regarding the optimisation results, Fig. 9 presents the evolution of the material parameters during the iteration process, the evaluations of the objective function required for the Jacobian matrix calculation are not included. Despite the optimisation process stopped just after 12 and 16 iterations depending on the initial set of parameters, most of the parameters reach a convergence plateau after 10 iterations. The evolution of the parameter $N$ shown in Fig. 9f, presents the most oscillating evolution. This fact can be caused by a lower sensitivity of the objective function to the parameter $N$.

Moreover, the identifications presented in this study were carried out with a standard computer, with an Intel(R) Core(TM) i7-6700 (2.60 GHz) processor and 16.00 GB of RAM memory. Depending on the initial set of parameters, the computational time is 57 seconds for the Sup_set and 86 seconds for the Inf_set. The computational time is significantly low, because it is used just one test in the virtual database and regarding that test, the number of time steps and the number of spatial points for the computation of internal work are reasonable. 


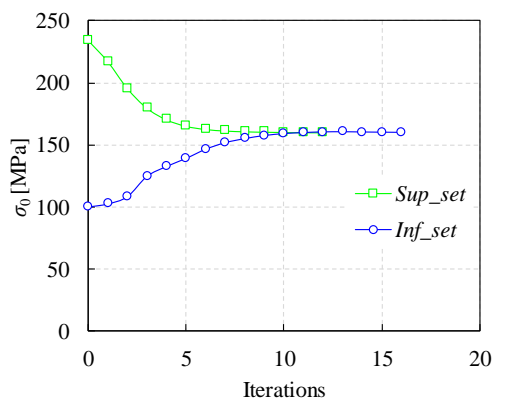

(a)

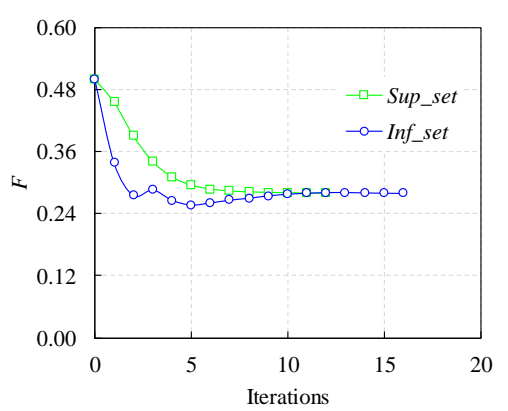

(d)

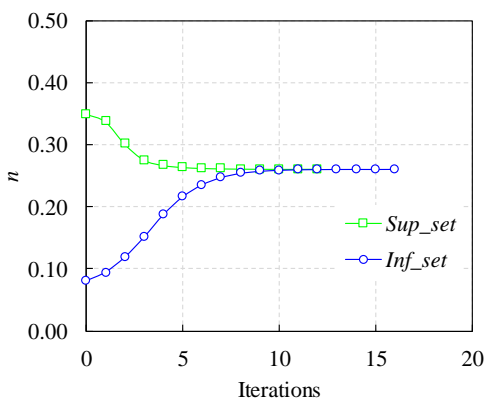

(b)

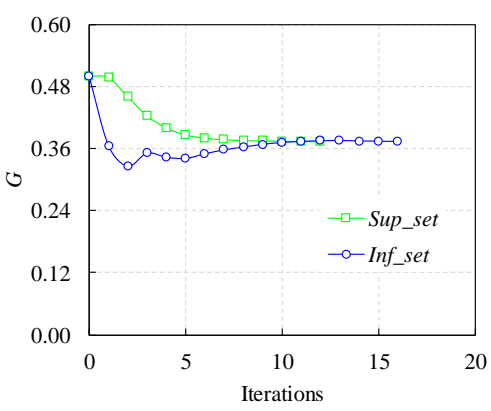

(e)

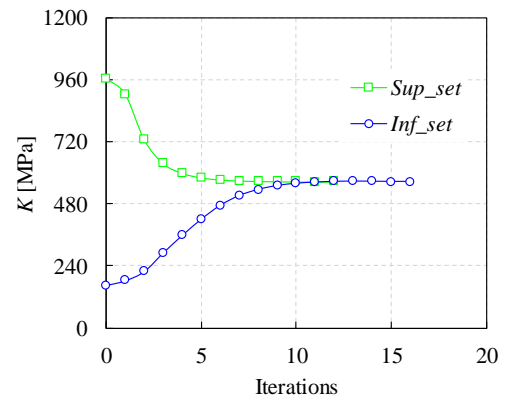

(c)

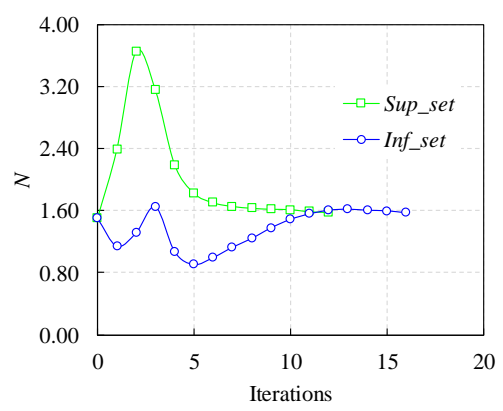

(f)

Figure 9: Evolution of the identified parameters for Cr3 during the optimisation process. 


\subsection{YLD2000-2d identification}

In this section, the combination of the VFM with the cruciform specimen is tested in a model with a more complex yield criterion, the YLD2000-2d. This yield criterion is combined with Swift's hardening law, resulting in a total of 11 parameters to be identified. Once again, the identification of the hardening and yield criterion parameters is performed simultaneously. The reference material parameters, which correspond to an aluminium alloy AA6016 in T4 state [42], are presented in Table 2. Cr3 provided the best results for the Hill'48 yield criterion, hence it is chosen for this analysis. The same FE model of Fig. 3c is used here. The simulation is displacement-driven, and $1 \mathrm{~mm}$ of displacement is applied to each arm of the specimen. This value is reduced in the present case to avoid exceeding the maximum force value. By means of a threedimensional model, it has been confirmed that after the maximum force value, the normal stress through thickness exhibits a sudden increase, which invalidates the plane stress condition and consequently, limits the application of the VFM beyond this point. The number of elements is increased up to 3226 to better describe the strain gradients developed in the arms of the specimen. The methodology adopted in the VFM procedure uses shape functions and the displacement fields extracted from the FE model at the nodes, to compute the strain tensor at the centroid of the element (as explained in section 2.1), which leads to an average strain tensor over the element. Thus, an increase of the number of elements mitigates the loss of information on the gradients present in the elements. In this case, an even finer mesh could be adopted, but no significant improvements in the results are noticed, whereas the computational time increases. Therefore, to have a computationally efficient mesh it is necessary to find a balance between identification results and computational time.

In this case, the integration of the constitutive model in the VFM is performed using a forward-Euler algorithm, which was also implemented in a UMAT subroutine for ABAQUS standard. This kind of integration scheme, despite requiring less computational time, requires a reduced increment size to prevent errors. Therefore, the number of time steps used in this simulation is 214 . These are also the number of time steps $n_{\mathrm{t}}$ used in the identification process. The equivalent plastic strain distribution for the last increment and the deformed FE mesh are presented in Fig. 10. It is possible to observe that deformation concentrates on the

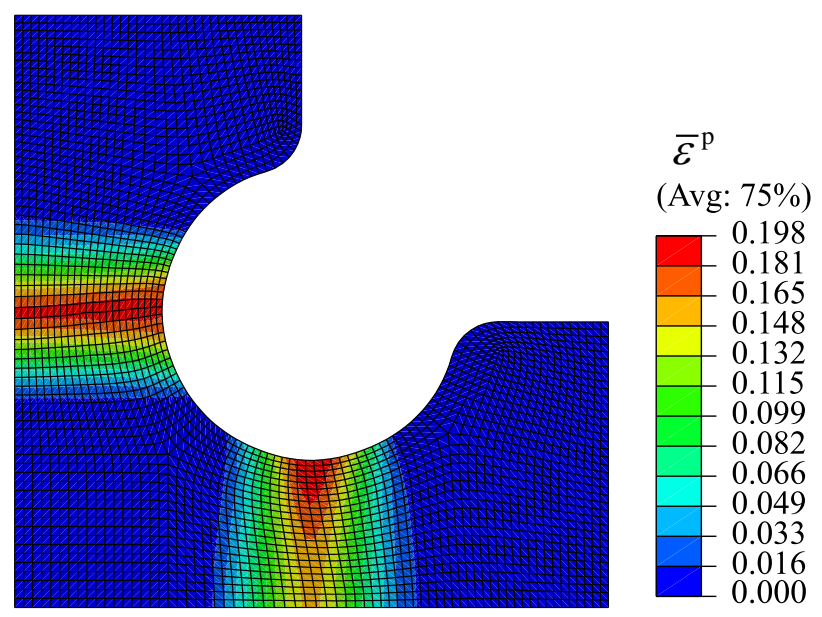

Figure 10: Equivalent plastic strain distribution for the last increment and the deformed FE mesh for Cr3 using the YLD2000-2d yield criterion and the material parameters of table 2 .

smallest section of the specimen. The maximum equivalent plastic strain value is around $\bar{\varepsilon}^{\mathrm{p}}=0.198$. The plot of the principal strains and stresses for the last increment is presented in Fig. 11. The equivalent plastic strain distributions and the principal strain plots (respectively Fig. 10 and Fig. 11a for the aluminium alloy and Fig. 3c and Fig. 4e for the mild steel) exhibit significant differences for the two materials. It comes from the very beginning of the test, due to a strong localisation in the arms for the aluminium alloy, that would also appear for the mild steel, if a large displacement was applied (above $4 \mathrm{~mm}$ ). Therefore, the strain paths close to the shear region shown in Fig. 3c can no longer be seen in Fig. 11a. This tendency to localize 


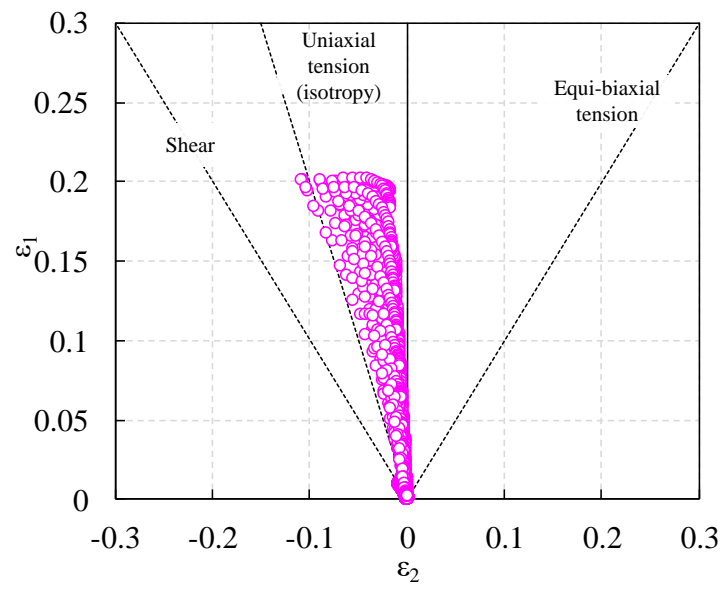

(a)

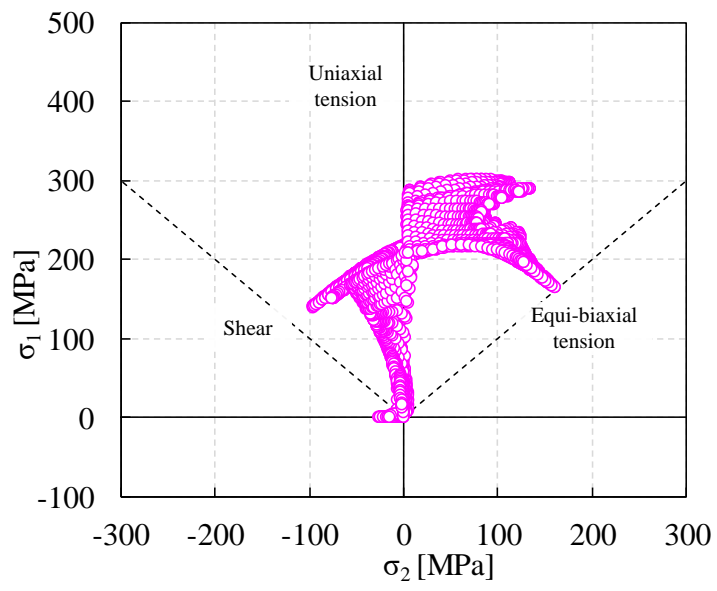

(b)

Figure 11: Plot of the strain (a) and stress (b) fields at the end of the test in the principal axes, corresponding to the model of Fig. 10.

is very strong for the aluminium alloy and starts almost at the beginning of plastic yielding. Indeed, for a maximum plastic strain of 0.01 , the principal strain plots for the aluminium alloy and mild steel display some similarities in the shear-tension quadrant, though, for the aluminium alloy, there are already strain states in the tension-plane strain quadrant, due to a tendency to strain localisation. It emphasises the fact that the design of a heterogeneous test for material parameter should be checked against an instability criterion to limit the range of deformation, to avoid excessive localisation. In order to test the stability of geometry Cr3, material parameters for YLD2000-2d for the mild steel have been identified from the plastic anisotropy coefficients and stress ratios given in Table 1, with a calculated biaxial coefficient [42]. A similar equivalent plastic strain distribution as in Fig. 3c is obtained. Moreover, for the aluminium alloy, two sets of Hill'48 coefficients have been identified, either from the plastic anisotropy coefficients or from the normalised stress ratios given in Table 4. As for the mild steel, the equivalent plastic strain distribution is close to the one obtained with YLD2000-2d model. These results highlight the fact that the test is more sensitive to the material than to the mechanical model. However, it is interesting to note that for the numerical simulation with Hill'48 model, with parameters identified from the stress ratios, the projection of the yield surface in the $\left(\sigma_{x x}, \sigma_{y y}\right)$ plane (material frame) is such that the plane strain point corresponds to a higher stress than the prediction based on Hill'48 model with parameters identified from the plastic anisotropy coefficients. This emphasises that the tendency to excessive localisation is also dependent on the model. It can be concluded that excessive localisation (or not) depends more significantly on the material than on the constitutive model.

As a consequence of the reduction of the applied displacement and strain localisation in the arms, a significant part of the stress points presented in Fig. 11b remains in the elastic regime or corresponds to a low value of equivalent plastic strain. The evolution of the resultant of the applied force for the two directions is presented in Fig. 12. The anisotropy effect is visible on the evolution of the force of the two arms. In this case, the force in the $y$-direction has the lowest value in the plastic regime.

As performed before, to check the presence of local minima in the objective function, two initial sets of parameters are tested which are presented in Table 5. The two sets differ on the values of the hardening parameters, whereas the $\alpha_{i}(\mathrm{i}=1, \ldots, 8)$ parameters always start from 1.0, corresponding to the isotropic case. The elastic parameters are considered to be known a priori. 


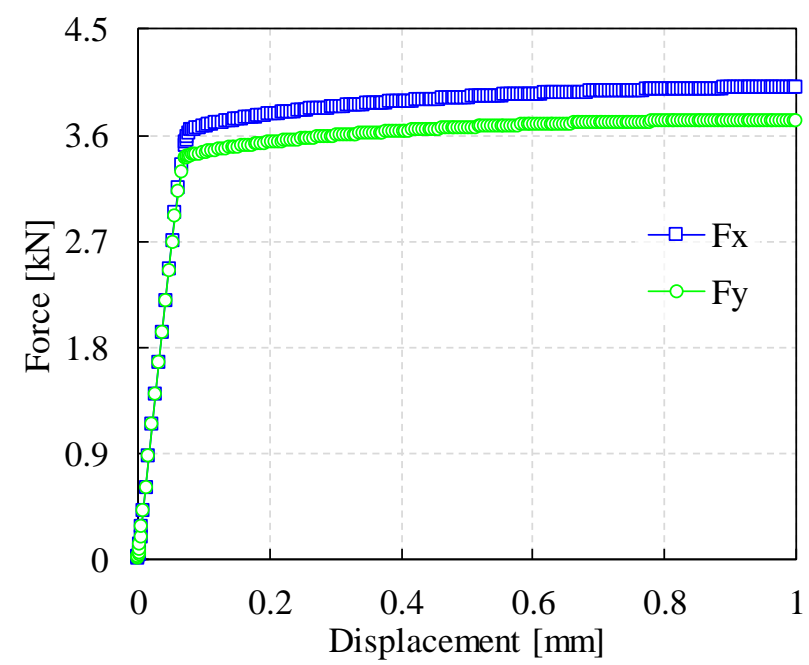

Figure 12: Force vs displacement for the two arms of the model of Fig 10.

\begin{tabular}{|c|c|c|c|c|c|c|c|c|c|}
\hline \multicolumn{4}{|c|}{ Swift's law } & & & & & & \\
\hline & $\sigma_{0}[\mathrm{MPa}]$ & $n$ & $K[\mathrm{MPa}]$ & & & & & & \\
\hline Reference & 212.03 & 0.239 & 385.47 & & & & & & \\
\hline Sup_set & 312 & 0.339 & 585 & & & & & & \\
\hline \multirow[t]{3}{*}{ Inf_set } & 112 & 0.139 & 185 & & & & & & \\
\hline & & YLD & 2000-2d yie & d criteri & & & & & \\
\hline & $\alpha_{1}$ & $\alpha_{2}$ & $\alpha_{3}$ & $\alpha_{4}$ & $\alpha_{5}$ & $\alpha_{6}$ & $\alpha_{7}$ & $\alpha_{8}$ & $m$ \\
\hline Reference & 0.9580 & 1.045 & 0.9485 & 1.0568 & 0.9938 & 0.9397 & 0.9200 & 1.1482 & 8.0 \\
\hline Sup_set \& Inf_set & 1.0 & 1.0 & 1.0 & 1.0 & 1.0 & 1.0 & 1.0 & 1.0 & 8.0 \\
\hline
\end{tabular}

Table 5: Reference parameters and initial parameter for Swift's law and YLD2000-2d yield criterion. 
The condition described by Eq. 26 is imposed on YLD200-2d, as a constraint added to the optimisation problem, by means of a penalty function. Therefore, the objective function becomes

$$
f=\varphi(\boldsymbol{\xi})+\delta \cdot \Im(\boldsymbol{\xi})
$$

where the parameter $\delta$ is a penalty coefficient which assumes the value $1 \times 10^{4}$. The function $\Im(\boldsymbol{\xi})$ can be written as

$$
\Im(\boldsymbol{\xi})=\left\{\left|\left[\frac{1}{2}\left(\left|\frac{2 \alpha_{1}+\alpha_{2}}{3}\right|^{a}+\left|\frac{2\left(\alpha_{3}-\alpha_{4}\right)}{3}\right|^{a}+\left|\frac{4 \alpha_{5}-\alpha_{6}}{3}\right|^{a}\right)\right]^{(1 / a)}-1\right|-\text { tol }\right\}^{2} .
$$

The parameter tol is a tolerance added to the constraint in order to prevent errors in this specific case, since using the reference parameters in Eq. 26 the value of 1 is not exactly reached. tol assumes the value of $1 \times 10^{-4}$. It should be mentioned that the absence of this condition leads to multiple solutions and its adoption reveals essential to avoid this problem. This particular behaviour of YLD2000-2d yield criterion was also reported by Güner et al. [41] and solved imposing the same constraint. Nevertheless, Güner et al. [41] imposed exactly the constraint of Eq. 26 which allowed to reduce the number of optimisation variables.

The results obtained with the constrained objective function are presented in Table 6 . The first thing to note is the fact that there is no sensitivity to the initial set of parameters, the two sets Sup_set and Inf_set converge on an identical solution, which suggests that the obtained set represents a global minimum. The results also show a good prediction of the initial yield stress $\sigma_{0}$, this is due to the constrained imposed. The remaining hardening parameters have higher errors, specially the hardening exponent. A plot of Swift's law for the reference and identified parameters as well as the force predicted based on the internal virtual work are presented in Fig. 13. The results for the force show a good match between the predicted and the reference (FE analysis). Moreover, despite the errors in the parameters $K$ and $n$, the reference and the identified Swift's law have a good match. Even when the results are extrapolated to $\bar{\varepsilon}^{\mathrm{p}}=0.50{ }^{1}$, there is only a slight overestimation of the flow stress.

For the yield criterion the highest errors are reached for the parameters $\alpha_{2}, \alpha_{8}$ and above all $\alpha_{7}$, the remaining have errors lower than $3 \%$. The errors in the parameters $\alpha_{7}$ and $\alpha_{8}$ follow a similar tendency as the results presented for Hill' 48 yield criterion, since these parameters are strictly connected to the shear components. This is coherent with the results presented in Fig. 11b, which show the lack of information on the fourth quadrant $\left(\sigma_{1}>0\right.$ and $\left.\sigma_{2}<0\right)$. This lack of data corresponds to a lower weight of the shear stress state in the optimisation. In order to better understand the quality of these identifications, the results of the predicted yield locus with the identified parameters (Sup_set) for different values of the ratio $\sigma_{x y} / \sigma_{0}$ in the normalised plane $\left(\sigma_{x x} / \sigma_{0}, \sigma_{y y} / \sigma_{0}\right)$ are presented in Fig. 14. The results show an accurate fit of the predicted yield locus for $\sigma_{x y} / \sigma_{0}=0.0$, revealing that the error of $4.7 \%$ in the parameter $\alpha_{2}$ has a minor influence on the results. Nevertheless, with the increase of $\sigma_{x y} / \sigma_{0}$, the identified parameters underestimate the form of the yield locus, a consequence of the overestimation of the parameters $\alpha_{7}$ and $\alpha_{8}$. Moreover, the predicted normalised yield stress and the plastic anisotropy coefficient $\left(r_{\alpha}\right)$ with the identified parameters for different angles of the tension axis are presented in Fig. 15. Similarly to the results obtained for Hill'48 yield criterion, the normalised yield stress is well predicted for $0^{\circ}$ and $90^{\circ}$, whereas it is underestimated for the remaining angles between $0^{\circ}$ and $90^{\circ}$. Regarding the prediction of the plastic anisotropy coefficient, there is a significant error and the tendency is to overestimate, with the exception of the angle $0^{\circ}$. These results for the plastic anisotropy coefficient can be explained by the intrinsic nature of the VFM. The method per se searches the best stress field for each step to minimise the difference between internal and external virtual works, whereas the plastic deformation prediction is a result of this process of minimisation.

Finally, the capacity of Cr3 to retrieve a large amount of material parameters, for YLD2000-2d criterion, 430 is also investigated for the mild steel. As previously mentioned, YLD2000-2d parameters are calculated from the plastic anisotropy coefficients and normalised yield stress ratios given in Table 1 and are presented in Table 7 as the reference values. It should be emphasised that the equivalent plastic strain distribution

\footnotetext{
${ }^{1}$ Note that the cruciform test only reaches a maximum value of equivalent plastic strain of $\bar{\varepsilon}^{\mathrm{p}}=0.19$
} 


\begin{tabular}{|c|c|c|c|c|c|c|c|c|}
\hline \multicolumn{4}{|c|}{ Swift's law } & & & & & \\
\hline & $\sigma_{0}[\mathrm{MPa}]$ & $n$ & $K[\mathrm{MPa}]$ & & & & & \\
\hline Reference & 212.03 & 0.239 & 385.47 & & & & & \\
\hline Sup_set & 212.40 & 0.260 & 398.07 & & & & & \\
\hline Error & $0.17 \%$ & $10.77 \%$ & $3.27 \%$ & & & & & \\
\hline Inf_set & 212.40 & 0.260 & 398.07 & & & & & \\
\hline Error & $0.17 \%$ & $10.77 \%$ & $3.27 \%$ & & & & & \\
\hline \multicolumn{9}{|c|}{ YLD2000-2d yield criterion $(\mathrm{m}=8)$} \\
\hline & $\alpha_{1}$ & $\alpha_{2}$ & $\alpha_{3}$ & $\alpha_{4}$ & $\alpha_{5}$ & $\alpha_{6}$ & $\alpha_{7}$ & $\alpha_{8}$ \\
\hline Reference & 0.9580 & 1.045 & 0.9485 & 1.0568 & 0.9938 & 0.9397 & 0.9200 & 1.1482 \\
\hline Sup_set & 0.9860 & 0.9959 & 0.9378 & 1.0647 & 0.9946 & 0.9491 & 1.0458 & 1.1963 \\
\hline Error & $2.92 \%$ & $4.70 \%$ & $1.13 \%$ & $0.75 \%$ & $0.08 \%$ & $1.00 \%$ & $13.67 \%$ & $4.19 \%$ \\
\hline Inf_set & 0.9860 & 0.9958 & 0.9378 & 1.0647 & 0.9956 & 0.9491 & 1.0459 & 1.1962 \\
\hline Error & $2.92 \%$ & $4.71 \%$ & $1.13 \%$ & $0.75 \%$ & $0.08 \%$ & $1.00 \%$ & $13.69 \%$ & $4.19 \%$ \\
\hline
\end{tabular}

Table 6: Results of the identification process for Swift's law and YLD2000-2d yield criterion.

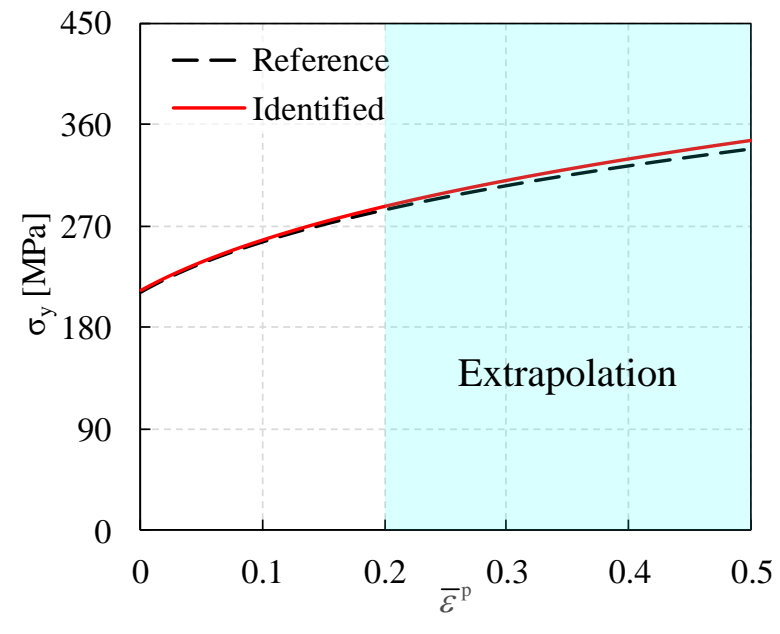

(a)

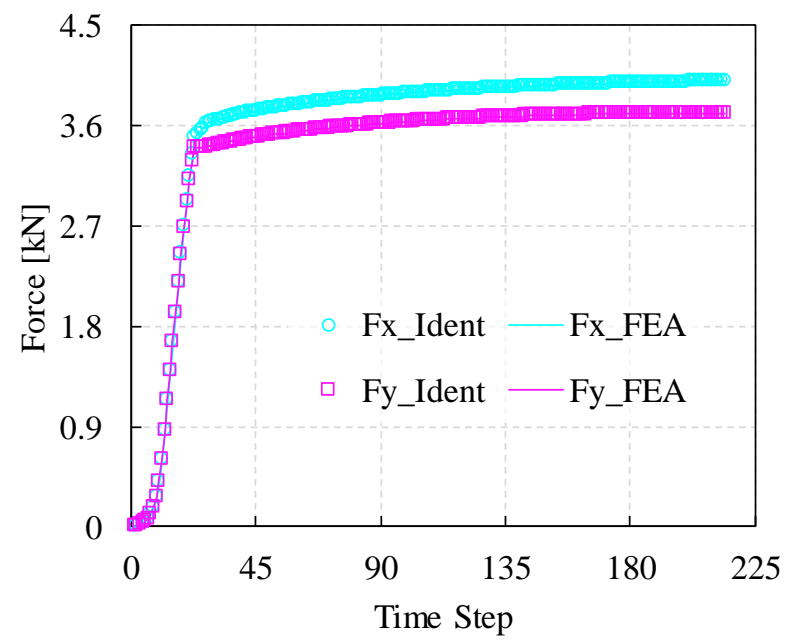

(b)

Figure 13: Results obtained with the identified parameter set Sup_set of Table 6: (a) plot of the Swift's law and (b) force prediction based on the internal work. 


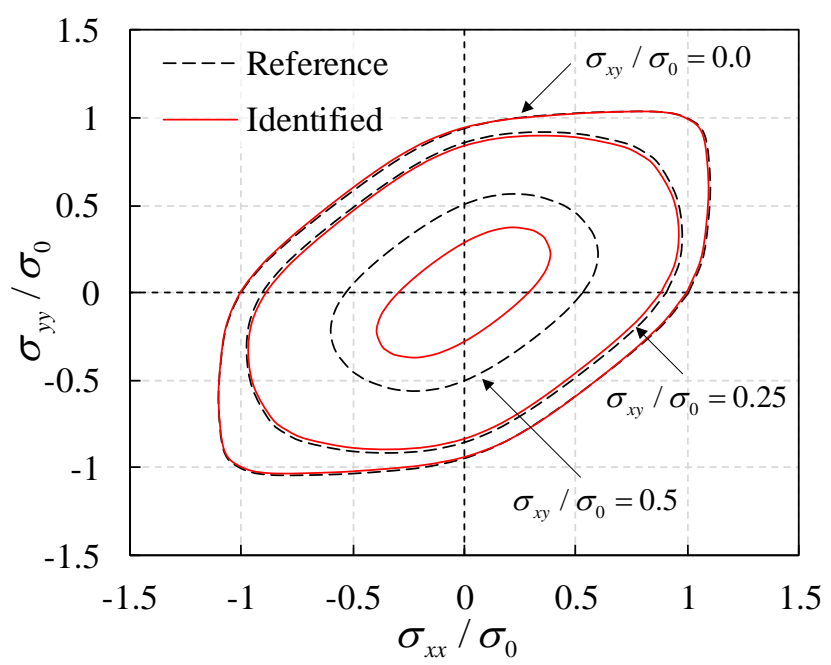

Figure 14: Comparison of the reference yield locus with the identified yield locus for different levels of $\sigma_{x y} / \sigma_{0}$.

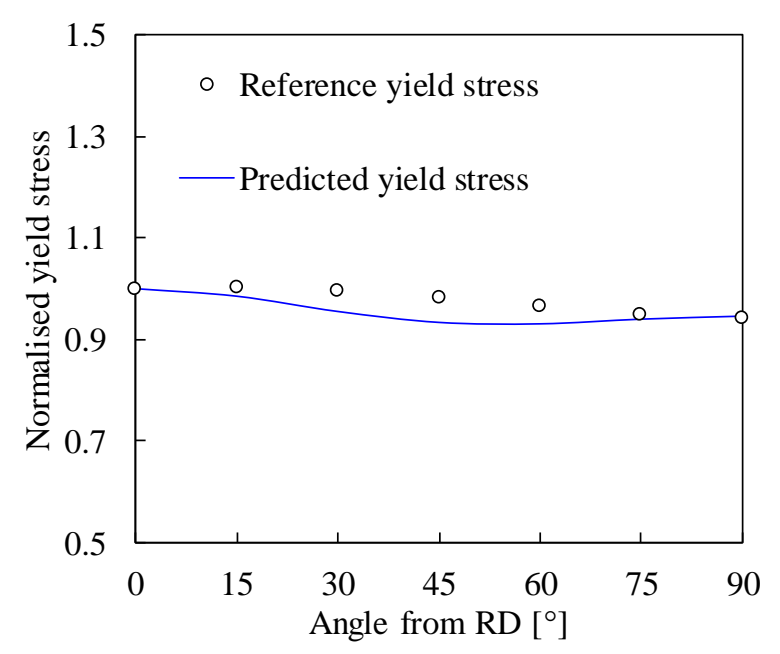

(a)

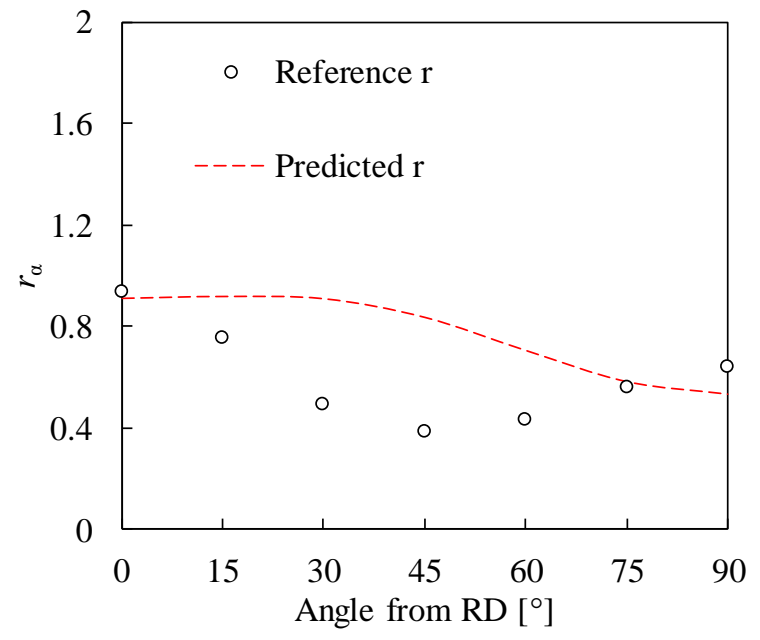

(b)

Figure 15: Evolution of the (a) normalised yield stress and (b) plastic anisotropy coefficient according to the tension axis angle from the rolling direction for the parameters identified with Cr3 and the aluminium alloy. 


\begin{tabular}{|c|c|c|c|c|c|c|c|c|}
\hline \multicolumn{4}{|c|}{ Swift's law } & & & & & \\
\hline & $\sigma_{0}[\mathrm{MPa}]$ & $n$ & $K[\mathrm{MPa}]$ & & & & & \\
\hline Reference & 160.0 & 0.26 & 565.0 & & & & & \\
\hline Sup_set & 159.87 & 0.265 & 571.51 & & & & & \\
\hline Error & $0.08 \%$ & $1.96 \%$ & $1.15 \%$ & & & & & \\
\hline Inf_set & 159.87 & 0.265 & 571.50 & & & & & \\
\hline Error & $0.08 \%$ & $1.96 \%$ & $1.15 \%$ & & & & & \\
\hline \multicolumn{9}{|c|}{ YLD2000-2d yield criterion $(\mathrm{m}=6)$} \\
\hline & $\alpha_{1}$ & $\alpha_{2}$ & $\alpha_{3}$ & $\alpha_{4}$ & $\alpha_{5}$ & $\alpha_{6}$ & $\alpha_{7}$ & $\alpha_{8}$ \\
\hline Reference & 1.0840 & 0.9459 & 0.7961 & 0.8739 & 0.9159 & 0.8048 & 1.0090 & 0.9822 \\
\hline Sup_set & 1.0831 & 0.9470 & 0.7955 & 0.8740 & 0.9150 & 0.8011 & 1.0088 & 0.9945 \\
\hline Error & $0.09 \%$ & $0.11 \%$ & $0.07 \%$ & $0.02 \%$ & $0.09 \%$ & $0.47 \%$ & $0.02 \%$ & $1.25 \%$ \\
\hline Inf_set & 1.0830 & 0.9470 & 0.7955 & 0.8741 & 0.9150 & 0.8011 & 1.0088 & 0.9945 \\
\hline Error & $0.09 \%$ & $0.11 \%$ & $0.08 \%$ & $0.02 \%$ & $0.10 \%$ & $0.47 \%$ & $0.02 \%$ & $1.25 \%$ \\
\hline
\end{tabular}

Table 7: Results of the identification process for Swift's law and YLD2000-2d yield criterion using the constitutive parameters for the mild steel.

and the stress and strain states in principal axes are very close to the ones presented in Figs. 3c, 4e and 4f. Therefore, the same procedure for the identification with the VFM is repeated. Two initial sets of parameters are used. The initial values for the hardening parameters are the ones presented in Table 2 and isotropic values are chosen for the yield criterion parameters. The results of these identifications are presented in Table 7 .

As can be concluded from Table 7 , the relative error in the retrieved material parameters is significantly lower compared to the aluminium alloy, specially for the parameters $\alpha_{2}, \alpha_{7}$ and, $\alpha_{8}$. The predicted normalised yield stresses and the plastic anisotropy coefficients $\left(r_{\alpha}\right)$ with the retrieved parameters (Sup_set) for different angles of the tension axis are presented in Fig. 16. The results show a very good agreement with the reference values. These results obtained for 2 materials show that the quality and richness of the information encoded in the heterogeneous strain fields provided by Cr3 leads to a very good prediction of material parameters for YLD2000-2d criterion, as long as the excessive strain localisation is not reached.

Concerning the computation time, it depends slightly on the initial set of parameters, respectively $944 \mathrm{~s}$ and $827 \mathrm{~s}$ for Sup_set and Inf_set. Comparing to the identification of the model composed by Hill' 48 yield criterion, there is an increase of one order of magnitude in the computational time. This can be attributed to the increase in the number of time steps and spatial integration points in the optimisation process. 


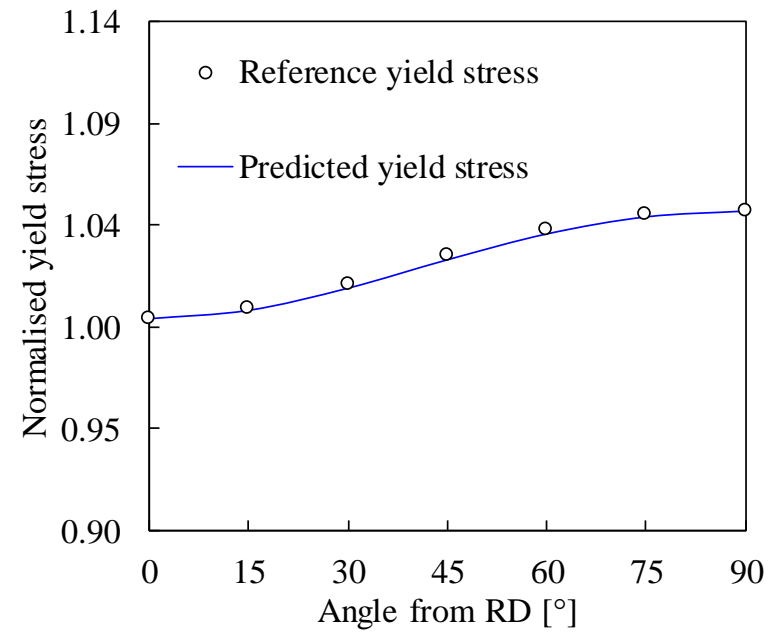

(a)

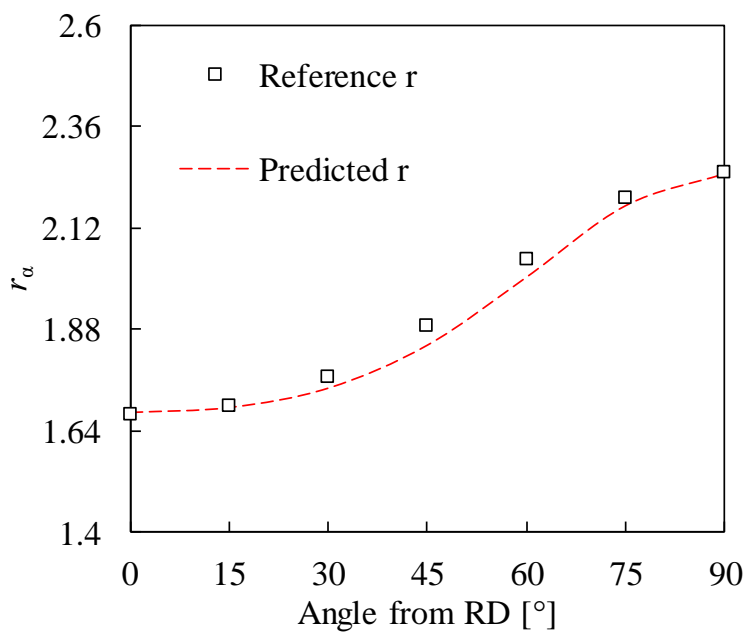

(b)

Figure 16: Evolution of (a) the normalised yield stress and (b) plastic anisotropy coefficient according to the tension axis angle from the rolling direction for the parameters identified with Cr3 and mild steel.

\section{Conclusion} test for the simultaneous identification of the para
hardening law using an the virtual fields method.

In a first step, three cruciform geometries are tested in the identification of the constitutive parameters of Hill'48 yield criterion and Swift's hardening law for a mild steel. The base for this study is the geomegeometrical adaptations to increase the strain and stress field heterogeneity. The comparison of the three geometries shows that the geometry proposed by Zhang et al. [16], despite providing a wide spread of stress states, lacks on information for shear stress states. This affects the identification of the yield criterion parameters, namely the parameter related with the shear component. Therefore, the increase of heterogeneity parameters of Hill'48 yield criterion. Thus, the other two geometries present better results, especially the third geometry which produces a wider dispersion of stress states and gives more information in the shear region. Moreover, all the identifications performed reached a single solution for the 6 parameters with a single test, independently of the initial set of parameters. Nevertheless, from this comparison it is concluded hat the third geometry provides the best results, with a maximum error of $1 \%$ for all the parameters

The second step addresses the identification of the material parameters of a more complex yield criterion, namely YLD2000-2d yield criterion, simultaneously with Swift's law. The third geometry analysed in the first step is chosen for this analysis. A reference set of constitutive parameters representing an aluminium alloy is selected. In this case, the objective function is modified to include a constraint on the YLD2000-2d criterion. In terms of information provided by the test, it is observed that the test is sensitive to the material used. The plastic behaviour of the aluminium alloy leads to strain localization in the smallest section of the specimen, which increases the information near plane strain, but reduces the information in the shear region. Nevertheless, the identification results prove to be insensitive to the initial set of parameters. The hardening curve is identified with reasonable accuracy, but the yield criterion parameters, specially the ones directly related with shear, contain high errors. A detailed analysis of the results shows that the error in the yield criterion parameters is not significant for the prediction of the initial yield stresses, but it has a more pronounced effect on the plastic anisotropy coefficient prediction. Moreover, a second identification 
is performed for the same model but with the parameters of the mild steel used in the first step. In this case, the identification results have a reasonable error (maximum error lower than $2 \%$ ), which emphasises the dependence on the chosen material.

In summary, the results of this second step show that is possible to identify all the material parameters from a constitutive model with a single test, but the accuracy of the identification is much dependent on the capability of the test to generate strain fields with relevant information. Moreover, it is highlighted that the information given by heterogeneous test can be dependent on the material selected and this can affect the accuracy of the identification results. Therefore, design methodologies of new heterogeneous tests should take into account the stability of the test when using different materials.

Finally, the computational cost should be highlighted. The combination of a single cruciform test, the VFM and the Levenberg-Marquardt method presented a reasonable computational time, difficult to achieve with other strategies, especially if compared with a FEMU-based inverse method. Even for a more complex model, such as YLD2000-2d yield criterion, the maximum time required for the simultaneous identification of all parameters was 944 seconds.

\section{Acknowledgements}

The authors gratefully acknowledge the financial support of the Portuguese Foundation for Science and Technology (FCT) under the projects CENTRO-01-0145-FEDER-029713, POCI-01-0145-FEDER-016876, POCI-01-0145-FEDER-031243 and POCI-01-0145-FEDER-030592 by UE/FEDER through the programs CENTRO 2020 and COMPETE 2020, and UID/EMS/00481/2013-FCT under CENTRO-01-0145-FEDER022083. The authors also would like to acknowledge the Région Bretagne (France) for its financial support. J.M.P. Martins is also grateful to the FCT for the PhD grant SFRH/BD/117432/2016.

\section{References}

[1] S. Bruschi, T. Altan, D. Banabic, P. Bariani, A. Brosius, J. Cao, A. Ghiotti, M. Khraisheh, M. Merklein, A. Tekkaya, Testing and modelling of material behaviour and formability in sheet metal forming, CIRP Annals 63 (2) (2014) 727-749. doi:http://dx.doi.org/10.1016/j.cirp.2014.05.005. URL http://linkinghub.elsevier.com/retrieve/pii/S0007850614001875

[2] M. Rabahallah, T. Balan, S. Bouvier, B. Bacroix, F. Barlat, K. Chung, C. Teodosiu, Parameter identification of advanced plastic strain rate potentials and impact on plastic anisotropy prediction, International Journal of Plasticity 25 (3) (2009) 491-512. doi:10.1016/j.ijplas.2008.03.006. URL http://linkinghub.elsevier.com/retrieve/pii/S0749641908000478

[3] K. Bandyopadhyay, K. Hariharan, M.-g. Lee, Q. Zhang, Robust multi objective optimization of anisotropic yield function coefficients, Materials \& Design 156 (2018) 184-197. doi:https://doi.org/10.1016/j.matdes.2018.06.033. URL https://linkinghub.elsevier.com/retrieve/pii/S0264127518305008

[4] S. Cooreman, D. Lecompte, H. Sol, J. Vantomme, D. Debruyne, Identification of Mechanical Material Behavior Through Inverse Modeling and DIC, Experimental Mechanics 48 (4) (2008) 421-433. doi:10.1007/s11340-007-9094-0. URL http://link.springer.com/10.1007/s11340-007-9094-0

[5] P. Prates, M. Oliveira, J. Fernandes, A new strategy for the simultaneous identification of constitutive laws parameters of metal sheets using a single test, Computational Materials Science 85 (2014) 102-120. doi:10.1016/j.commatsci.2013. 12.043 . URL http://linkinghub.elsevier.com/retrieve/pii/S0927025613008021

[6] J. Réthoré, Muhibullah, T. Elguedj, M. Coret, P. Chaudet, A. Combescure, Robust identification of elasto-plastic constitutive law parameters from digital images using 3D kinematics, International Journal of Solids and Structures 50 (1) (2013) 73-85. doi:10.1016/j.ijsolstr.2012.09.002. URL http://linkinghub.elsevier.com/retrieve/pii/S0020768312003836

[7] H. W. Swift, Plastic instability under plane stress, Journal of the Mechanics and Physics of Solids 1 (1) (1952) 1-18. doi:10.1016/0022-5096(52)90002-1.

URL https://www.sciencedirect.com/science/article/pii/0022509652900021http://linkinghub.elsevier.com/ retrieve/pii/0022509652900021

[8] N. Tardif, S. Kyriakides, Determination of anisotropy and material hardening for aluminum sheet metal, International Journal of Solids and Structures 49 (25) (2012) 3496-3506. doi:10.1016/j.ijsolstr.2012.01.011. URL https://www.sciencedirect.com/science/article/pii/S0020768312000273http://linkinghub.elsevier.com/ retrieve/pii/S0020768312000273 
[9] R. Hill, A theory of the yielding and plastic flow of anisotropic metals, Proceedings of the Royal Society of London A: Mathematical, Physical and Engineering Sciences 193 (1033) (1948) 281-297. arXiv:http://rspa.royalsocietypublishing. org/content/193/1033/281.full.pdf, doi:10.1098/rspa.1948.0045. URL http://rspa.royalsocietypublishing.org/content/193/1033/281

[10] R. Pearce, Some aspects of anisotropic plasticity in sheet metals, International Journal of Mechanical Sciences 10 (12) (1968) 995 - 1004. doi:https://doi.org/10.1016/0020-7403(68)90053-2. URL http://www.sciencedirect.com/science/article/pii/0020740368900532

[11] D. Banabic, Plastic Behaviour of Sheet Metal, Springer Berlin Heidelberg, Berlin, Heidelberg, 2010, pp. 27-140. doi: 10.1007/978-3-540-88113-1_2. URL https://doi.org/10.1007/978-3-540-88113-1_2

540 [12] F. Barlat, J. Brem, J. Yoon, K. Chung, R. Dick, D. Lege, F. Pourboghrat, S.-H. Choi, E. Chu, Plane stress yield function for aluminum alloy sheets - part I: theory, International Journal of Plasticity 19 (9) (2003) 1297 - 1319. doi:https: //doi.org/10.1016/S0749-6419(02)00019-0.

URL http://www.sciencedirect.com/science/article/pii/S0749641902000190

[13] S. Avril, M. Bonnet, A.-S. Bretelle, M. Grédiac, F. Hild, P. Ienny, F. Latourte, D. Lemosse, S. Pagano, E. Pagnacco, F. Pierron, Overview of Identification Methods of Mechanical Parameters Based on Full-field Measurements, Experimental Mechanics 48 (4) (2008) 381-402. doi:10.1007/s11340-008-9148-y. URL http://link.springer.com/10.1007/s11340-008-9148-y

[14] J. M. Martins, A. Andrade-Campos, S. Thuillier, Comparison of inverse identification strategies for constitutive mechanical models using full-field measurements, International Journal of Mechanical Sciences 145 (February) (2018) 330-345. doi: 10.1016/j.ijmecsci.2018.07.013. URL https://doi.org/10.1016/j.ijmecsci.2018.07.013

[15] P. A. Prates, A. F. G. Pereira, N. A. Sakharova, M. C. Oliveira, J. V. Fernandes, Inverse Strategies for Identifying the Parameters of Constitutive Laws of Metal Sheets, Advances in Materials Science and Engineering 2016 (Dic) (2016) 1-18. doi:10.1155/2016/4152963 URL https://www.hindawi.com/journals/amse/2016/4152963/

[16] S. Zhang, L. Leotoing, D. Guines, S. Thuillier, S. lai Zang, Calibration of anisotropic yield criterion with conventional tests or biaxial test, International Journal of Mechanical Sciences 85 (2014) $142-151$. doi:https://doi.org/10.1016/j. ijmecsci.2014.05.020.

[17] N. Souto, S. Thuillier, A. Andrade-Campos, Design of an indicator to characterize and classify mechanical tests for sheet metals, International Journal of Mechanical Sciences 101-102 (2015) $252-271$. doi:https://doi.org/10.1016/j. ijmecsci.2015.07.026.

[18] F. Bron, J. Besson, A yield function for anisotropic materials application to aluminum alloys, International Journal of Plasticity 20 (4) (2004) 937 - 963. doi:https://doi.org/10.1016/j.ijplas.2003.06.001.

[19] S. Coppieters, T. Hakoyama, D. Debruyne, S. Takahashi, T. Kuwabara, Inverse Yield Locus Identification using a biaxial tension apparatus with link mechanism and displacement fields, Journal of Physics: Conference Series 1063 (2018) 012039. doi : 10.1088/1742-6596/1063/1/012039. URL http://stacks.iop.org/1742-6596/1063/i=1/a=012039?key=crossref .a1cd34c8f130c81243a079bd967c9abf

[20] D. Lecompte, A. Smits, H. Sol, J. Vantomme, D. V. Hemelrijck, Mixed numericalexperimental technique for orthotropic parameter identification using biaxial tensile tests on cruciform specimens, International Journal of Solids and Structures 44 (5) (2007) 1643 - 1656. doi:https://doi.org/10.1016/j.ijsolstr.2006.06.050.

[21] F. Pierron, M. Grédiac, The virtual fields method: extracting constitutive mechanical parameters from full-field deformation measurements, Springer Science \& Business Media, 2012.

[22] M. Rossi, F. Pierron, M. Štamborská, Application of the virtual fields method to large strain anisotropic plasticity, International Journal of Solids and Structures 97-98 (2016) 322-335. doi:https://doi.org/10.1016/j.ijsolstr.2016. 07.015 . URL http://www.sciencedirect.com/science/article/pii/S0020768316301755

[23] A. Marek, F. M. Davis, M. Rossi, F. Pierron, Extension of the sensitivity-based virtual fields to large deformation anisotropic plasticity, International Journal of Material Formingdoi:10.1007/s12289-018-1428-1. URL https://doi.org/10.1007/s12289-018-1428-1

[24] J. Fu, F. Barlat, J.-H. Kim, F. Pierron, Identification of nonlinear kinematic hardening constitutive model parameters using the virtual fields method for advanced high strength steels, International Journal of Solids and Structures $102-103$ (2016) $30-43$. doi:https://doi.org/10.1016/j.ijsolstr.2016.10.020.

[25] J. Fu, F. Barlat, J. H. Kim, F. Pierron, Application of the virtual fields method to the identification of the homogeneous anisotropic hardening parameters for advanced high strength steels, International Journal of Plasticity 93 (2017) $229-250$. doi: 10.1016/j.ijplas.2016.07.013. URL https://www.sciencedirect.com/science/article/pii/S074964191630122X

[26] N. Promma, B. Raka, M. Grédiac, E. Toussaint, J.-B. L. Cam, X. Balandraud, F. Hild, Application of the virtual fields method to mechanical characterization of elastomeric materials, International Journal of Solids and Structures 46 (3) (2009) 698 - 715. doi:https://doi.org/10.1016/j.ijsolstr.2008.09.025. URL http://www.sciencedirect.com/science/article/pii/S0020768308003946

[27] M. Rossi, F. Pierron, Identification of plastic constitutive parameters at large deformations from three dimensional displacement fields, Computational Mechanics 49 (1) (2012) 53-71. doi:10.1007/s00466-011-0627-0. URL https://doi.org/10.1007/s00466-011-0627-0

[28] F. Pierron, S. Avril, V. T. Tran, Extension of the virtual fields method to elasto-plastic material identification with cyclic 
loads and kinematic hardening, International Journal of Solids and Structures 47 (22) (2010) 2993 - 3010. doi:https: //doi.org/10.1016/j.ijsolstr.2010.06.022.

[29] D. Notta-Cuvier, B. Langrand, F. Lauro, E. Markiewicz, An innovative procedure for characterising a coupled elastoplastic damage model of behaviour using the virtual fields method, International Journal of Solids and Structures 69-70 (2015) 415 - 427. doi:https://doi.org/10.1016/j.ijsolstr.2015.05.009. URL http://www.sciencedirect.com/science/article/pii/S002076831500219X

[30] A. G. Holzapfel, Nonlinear Solid Mechanics: A Continuum Approach for Engineering, John Wiley \& Sons, LTD., 2000.

[31] M. Rossi, L. Cortese, K. Genovese, A. Lattanzi, F. Nalli, F. Pierron, Evaluation of volume deformation from surface dic measurement, Experimental Mechanicsdoi:10.1007/s11340-018-0409-0. URL https://doi.org/10.1007/s11340-018-0409-0

605 [32] B. Rahmani, E. Ghossein, I. Villemure, M. Levesque, In-situ mechanical properties identification of 3d particulate composites using the virtual fields method, International Journal of Solids and Structures 51 (18) (2014) 3076 - 3086. doi:https://doi.org/10.1016/j.ijsolstr.2014.05.006.

URL http://www.sciencedirect.com/science/article/pii/S0020768314001929

[33] J. N. Reddy, An Introduction to Continuum Mechanics, 2nd Edition, Cambridge University Press, 2013. doi:10.1017/ CB09781139178952.

[34] M. Crisfield, Non-Linear Finite Element Analysis of Solids and Structures, Volume 1, John Wiley \& Sons, Ltd., 1991.

[35] J. C. Simo, T. J. R. Hughes, Computational inelasticity, Springer, New York, NY, 1998. doi:https://doi.org/10.1007/ b98904.

[36] A. Marek, F. M. Davis, F. Pierron, Sensitivity-based virtual fields for the non-linear virtual fields method, Computational Mechanics 60 (3) (2017) 409-431. doi:10.1007/s00466-017-1411-6. URL http://link.springer.com/10.1007/s00466-017-1411-6

[37] J.-H. Kim, F. Barlat, F. Pierron, M.-G. Lee, Determination of anisotropic plastic constitutive parameters using the virtual fields method, Experimental Mechanics 54 (7) (2014) 1189-1204. doi:10.1007/s11340-014-9879-x. URL https://doi.org/10.1007/s11340-014-9879-x

[38] D. W. Marquardt, An algorithm for least-squares estimation of nonlinear parameters, Journal of the society for Industrial and Applied Mathematics 11 (2) (1963) 431-441. doi:10.1137/0111030.

[39] O. Cazacu, B. Revil-Baudard, N. Chandola, Plasticity-Damage Couplings: From Single Crystal to Polycrystalline Materials, Vol. 253, Springer, 2019. doi:10.1007/978-3-319-92922-4.

URL http://link. springer.com/10.1007/978-3-319-92922-4

[40] J.-H. Kim, A. Serpantié, F. Barlat, F. Pierron, M.-G. Lee, Characterization of the post-necking strain hardening behavior using the virtual fields method, International Journal of Solids and Structures 50 (24) (2013) 3829-3842. doi:10.1016/j. ijsolstr.2013.07.018.

URL http://linkinghub.elsevier.com/retrieve/pii/S0020768313002989

[41] A. Guner, C. Soyarslan, A. Brosius, A. Tekkaya, Characterization of anisotropy of sheet metals employing inhomogeneous strain fields for Yld2000-2D yield function, International Journal of Solids and Structures 49 (25) (2012) 3517 - 3527. doi:https://doi.org/10.1016/j.ijsolstr.2012.05.001. URL http://www.sciencedirect.com/science/article/pii/S0020768312001928

[42] J.-W. Yoon, F. Barlat, R. E. Dick, K. Chung, T. J. Kang, Plane stress yield function for aluminum alloy sheets - part II: Fe formulation and its implementation, International Journal of Plasticity 20 (3) (2004) 495 - 522, Owen Richmond Memorial Special Issue. doi:https://doi.org/10.1016/S0749-6419(03)00099-8 URL http://www.sciencedirect.com/science/article/pii/S0749641903000998

[43] T. Pottier, F. Toussaint, P. Vacher, Contribution of heterogeneous strain field measurements and boundary conditions modelling in inverse identification of material parameters, European Journal of Mechanics - A/Solids 30 (3) (2011) 373 382. doi:https://doi.org/10.1016/j.euromechsol.2010.10.001.

[44] S. Schmaltz, K. Willner, Comparison of different biaxial tests for the inverse identification of sheet steel material parameters, Strain 50 (5) (2014) 389-403. doi:10.1111/str.12080. URL https://onlinelibrary.wiley.com/doi/abs/10.1111/str.12080

[45] K. Denys, S. Coppieters, M. Seefeldt, D. Debruyne, Multi-DIC setup for the identification of a 3d anisotropic yield surface of thick high strength steel using a double perforated specimen, Mechanics of Materials 100 (2016) 96 - 108. doi:https://doi.org/10.1016/j.mechmat.2016.06.011. URL http://www.sciencedirect.com/science/article/pii/S0167663616300989

[46] J. M. P. Martins, S. Thuillier, A. Andrade-Campos, Identification of material parameters for plasticity models: A comparative study on the finite element model updating and the virtual fields method, AIP Conference Proceedings 1960 (1) (2018) 110007. doi:10.1063/1.5034964. URL https://aip.scitation.org/doi/abs/10.1063/1.5034964

[47] E. Jones, J. Carroll, K. Karlson, S. Kramer, R. Lehoucq, P. Reu, D. Turner, Parameter covariance and non-uniqueness in material model calibration using the virtual fields method, Computational Materials Science 152 (2018) 268 - 290. doi:https://doi.org/10.1016/j.commatsci.2018.05.037. URL http://www.sciencedirect.com/science/article/pii/S0927025618303501 